\title{
LOCAL UNITARY PERIODS AND RELATIVE DISCRETE SERIES
}

\author{
JERROD MANFORD SMITH
}

\begin{abstract}
Let $F$ be a $p$-adic field $(p \neq 2)$, let $E$ be a quadratic Galois extension of $F$, and let $n \geq 2$. We construct representations in the discrete spectrum of the $p$-adic symmetric space $H \backslash G$, where $G=\mathbf{G L}_{2 n}(E)$ and $H=\mathbf{U}_{E / F}(F)$ is a quasi-split unitary group over $F$.
\end{abstract}

\section{Contents}

1. Introduction

2. Notation and preliminaries

3. Background on RDS: the Relative Casselman's Criterion 7

4. $p$-adic symmetric spaces and parabolic subgroups 9

5. Relative discrete series for $\mathbf{U}_{E / F}(F) \backslash \mathbf{G L}_{2 n}(E) \quad 18$

6. A technical lemma 27

Acknowledgements $\quad 29$

References $\quad 29$

\section{INTRODUCTION}

Let $F$ be a $p$-adic field $(p \neq 2)$ and let $E$ be a quadratic Galois extension of $F$. Let $G=\mathbf{G L}_{n}(E)$ and let $H$ be the group of $F$-points of a quasisplit unitary group $\mathbf{U}_{E / F}$. In this paper, we are concerned with constructing irreducible representations of $G$ that occur in the discrete spectrum ${ }^{1}$ $L_{\text {disc }}^{2}(H \backslash G)$ of the $p$-adic symmetric space $H \backslash G$. Such representations are referred to as relative discrete series (RDS) representations for $H \backslash G$. Of course, the issue of characterizing the discrete spectrum is of interest more

Date: June 21, 2018.

2010 Mathematics Subject Classification. Primary 22E50; Secondary 22E35.

Key words and phrases. Relative discrete series, $p$-adic symmetric space, distinguished representation, unitary period, quasi-split unitary group.

The author was partially supported by the NSERC, Canada Graduate Scholarship and the Ontario Graduate Scholarship programs.

${ }^{1}$ To make sense of $L_{\text {disc }}^{2}(H \backslash G)$, one also needs to take the quotient by the centre $Z_{G}$ of $G$ and consider square integrable functions on the quotient $Z_{G} H \backslash G$. Moreover, we must consider representations that admit a (unitary) central character. 
generally. One would eventually strive for a recipe to construct representations in $L_{\text {disc }}^{2}(H \backslash G)$ for any connected reductive group $\mathbf{G}$ and symmetric subgroup $\mathbf{H}=\mathbf{G}^{\theta}$, where $\theta$ is an $F$-involution of $\mathbf{G}$. For the symmetric spaces $\mathbf{G L}_{n}(F) \times \mathbf{G L}_{n}(F) \backslash \mathbf{G} \mathbf{L}_{2 n}(F)$ and $\mathbf{G} \mathbf{L}_{n}(F) \backslash \mathbf{G} \mathbf{L}_{n}(E)$, the author has carried out a construction of RDS, analogous to the one in Theorem 5.11, in [29, Theorem 6.3]. Sakellaridis and Venkatesh have considered (and answered) much more general questions in the harmonic analysis on $p$-adic spherical varieties in [28; however, they do not give an explicit description of the discrete spectrum. Understanding the discrete series for $p$-adic symmetric spaces is a natural first step towards the general picture for spherical varieties. Moreover, it is known by work of Kato and Takano [21, that $H$ distinguished ${ }^{2}$ discrete series representations of $G$ are automatically RDS. We are thus interested in constructing RDS that do not occur in the discrete spectrum of $G$.

In the case of number fields, distinction by unitary groups, that is, nonvanishing of the period integral attached to $\mathbf{U}_{E / F}$, has deep connections with quadratic base change [1. The study of global period integrals has largely been pioneered by H. Jacquet and his collaborators (see, for instance, [15, 16, 17, 18, 19]). We refer the reader to the work of Feigon, Lapid and Offen [8] for a detailed discussion of both local and global aspects of the theory, as well as a very nice treatment of the history of the subject and the contributions of $\mathrm{H}$. Jacquet, et al. We recall some of the results of [8] in Section 5.1.1; however, we will only state those results that we require to prove our main theorem. We encourage the reader to consult the work of Feigon, Lapid and Offen for more details; including a discussion of the failure of local multiplicity-one [8, §13].

We now give a statement of the main result of the paper. Let $n \geq 2$ be an integer. Let $Q=P_{(n, n)}$ be the upper-triangular parabolic subgroup of $\mathbf{G L}_{2 n}(E)$, with standard Levi factorization $L=M_{(n, n)}, U=N_{(n, n)}$.

Theorem Theorem 5.11. Let $\pi=\iota_{Q}^{G} \tau$ be a parabolically induced representation, where $\tau=\tau^{\prime} \otimes{ }^{\sigma} \tau^{\prime}$, and $\tau^{\prime}$ is a discrete series representation of $\mathbf{G L}_{n}(E)$ such that $\tau^{\prime}$ is not Galois invariant, i.e., $\tau^{\prime} ¥{ }^{\sigma} \tau^{\prime}$. The representation $\pi$ is a relative discrete series representation for $\mathbf{U}_{E / F}(F) \backslash \mathbf{G L}_{2 n}(E)$ that does not occur in the discrete series of $\mathbf{G L}_{2 n}(E)$.

Theorem 5.11 is the direct analogue of [29, Theorem 6.3] and the overall method of proof is the same. The idea of the proof is to reduce the verification of the Relative Casselman's Criterion (Theorem 3.4) of Kato and Takano to the usual Casselman's Criterion for the inducing discrete series. In contrast to the work in [29, here we can construct representations only by inducing from the maximal parabolic subgroup $P_{(n, n)}$ due to the nature of the symmetric space $H \backslash G$ and the (lack of) existence of $\theta$-elliptic Levi

\footnotetext{
${ }^{2}$ See Definition 2.4
} 
subgroups ( $c f$. Definition 4.4, Lemma 4.20). Similarly, the lack of $\theta$-elliptic Levi subgroups prevents us from considering $\mathbf{G L}_{n}(E)$, when $n$ is odd.

Remark 1.1. In light of recent work of Raphaël Beuzart-Plessis, announced in his 2017 Cours Peccot, our construction of RDS via Theorem 5.11 exhausts all relative discrete series for the symmetric pair $\mathbf{U}_{E / F}(F) \backslash \mathbf{G L}_{2 n}(E)$ that lie outside of $L_{\text {disc }}^{2}(G)$. The remaining relative discrete series are the $\mathbf{U}_{E / F}(F)$-distinguished discrete series representations of $\mathbf{G L}_{2 n}(E)$. We discuss the exhaustion of the discrete spectrum in Section 5.4.

We also prove the following corollary to Theorem 5.11.

Corollary Corollary 5.13. Let $n \geq 4$ be an integer that is not an odd prime. There exist infinitely many equivalence classes of $R D S$ representations of the form constructed in Theorem 5.11 and such that the discrete series $\tau$ is not supercuspidal.

We now give an outline of the contents of the paper. In Section 2, we set notation and recall basic facts regarding parabolic induction and distinguished representations. We review the Relative Casselman's Criterion, due to Kato and Takano [21, in Section 3. We also discuss the invariant forms on Jacquet modules constructed by Kato and Takano [20], and Lagier 22. In Section 4, we recall the required structural results on $p$-adic symmetric spaces. In particular, we discuss $(\theta, F)$-split tori, $\theta$-split parabolic subgroups, $\theta$-elliptic Levi subgroups, and the relative root system. The main results of the paper (Theorem 5.11 and Corollary 5.13) are stated and proved in Section 5. In Section 6 we prove the remaining technical results needed to establish the main theorem.

\section{NotATION AND PRELIMINARIES}

Let $F$ be a nonarchimedean local field of characteristic zero and odd residual characteristic. Let $\mathcal{O}_{F}$ be the ring of integers of $F$. Let $E$ be a quadratic Galois extension of $F$. Let $\sigma \in \operatorname{Gal}(E / F)$ the nontrivial element of the Galois group of $E$ over $F$.

For now, let $\mathbf{G}$ be an arbitrary connected reductive group defined over $F$ and let $G=\mathbf{G}(F)$ denote the group of $F$-points. We will restrict to the case that $G=\mathbf{G L}_{n}(E)$ in Section 5 (onwards). We let $Z_{G}$ denote the centre of $G$, and let $A_{G}$ denote the $F$-split component of $Z_{G}$. Let $\theta$ be an $F$-involution of $\mathbf{G}$. Define $\mathbf{H}=\mathbf{G}^{\theta}$ to be the (closed) subgroup of $\theta$-fixed points of $\mathbf{G}$. The quotient $H \backslash G$ is a $p$-adic symmetric space.

We will routinely abuse notation and identify an algebraic group defined over $F$ with its group of $F$-points. When the distinction is to be made, we will use boldface to denote the algebraic group and regular typeface to denote the group of $F$-points. For any $F$-torus $\mathbf{A}$, we let $A^{1}$ denote the group of $\mathcal{O}_{F}$-points of $\mathbf{A}$.

Let $\mathbf{G L}_{n}$ denote the general linear group of $n$ by $n$ invertible matrices. We write $\mathbf{P}_{(\underline{m})}$ for the block-upper triangular parabolic subgroup of $\mathbf{G L}_{n}$, 
corresponding to a partition $(\underline{m})=\left(m_{1}, \ldots, m_{k}\right)$ of $n$. The group $\mathbf{P}_{(\underline{m})}$ has block-diagonal Levi subgroup $\mathbf{M}_{(\underline{m})} \cong \prod_{i=1}^{k} \mathbf{G L}_{m_{i}}$ and unipotent radical $\mathbf{N}_{(\underline{m})}$. We use $\operatorname{diag}\left(a_{1}, a_{2}, \ldots, a_{n}\right)$ to denote an $n \times n$ diagonal matrix with entries $a_{1}, \ldots, a_{n}$.

For any $g, x \in G$, we write ${ }^{g} x=g x g^{-1}$. For any subset $X$ of $G$, we write ${ }^{g} X=\left\{{ }^{g} x: x \in X\right\}$. Let $C_{G}(X)$ denote the centralizer of $X$ in $G$ and let $N_{G}(X)$ be the normalizer of $X$ in $G$. Given a real number $r$ we let $\lfloor r\rfloor$ denote the greatest integer that is less than or equal to $r$. We use $\widehat{(\cdot)}$ to denote that a symbol is omitted. For instance, $\operatorname{diag}\left(\widehat{a_{1}}, a_{2}, \ldots, a_{n}\right)$ may be used to denote the diagonal matrix $\operatorname{diag}\left(a_{2}, \ldots, a_{n}\right)$.

2.1. Induced representations of $p$-adic groups. We now briefly review some necessary background of the representation theory of $G$ and discuss the representations that are relevant in the harmonic analysis on $H \backslash G$. We will only consider representations on complex vector spaces. A representation $(\pi, V)$ of $G$ is smooth if for every $v \in V$ the stabilizer of $v$ in $G$ is an open subgroup. A smooth representation $(\pi, V)$ of $G$ is admissible if, for every compact open subgroup $K$ of $G$, the subspace $V^{K}$ of $K$-invariant vectors is finite dimensional. All of the representations that we consider are smooth and admissible. A quasi-character of $G$ is a one-dimensional representation. Let $(\pi, V)$ be a smooth representation of $G$. If $\omega$ is a quasi-character of $Z_{G}$, then $(\pi, V)$ is an $\omega$-representation if $\pi$ has central character $\omega$.

Let $P$ be a parabolic subgroup of $G$ with Levi subgroup $M$ and unipotent radical $N$. Given a smooth representation $\left(\rho, V_{\rho}\right)$ of $M$ we may inflate $\rho$ to a representation of $P$, also denoted $\rho$, by declaring that $N$ acts trivially. We define the representation $\iota_{P}^{G} \rho$ of $G$ to be the (normalized) parabolically induced representation $\operatorname{Ind}_{P}^{G}\left(\delta_{P}^{1 / 2} \otimes \rho\right)$. We normalize by the square root of the modular character $\delta_{P}$ of $P$. The character $\delta_{P}$ is given by $\delta_{P}(p)=$ $\left|\operatorname{det} \operatorname{Ad}_{\mathfrak{n}}(p)\right|$, for all $p \in P$, where $\operatorname{Ad}_{\mathfrak{n}}$ denotes the adjoint action of $P$ on the Lie algebra $\mathfrak{n}$ of $N$ [6]. Let $(\pi, V)$ be a smooth representation of $G$. Let $\left(\pi_{N}, V_{N}\right)$ denote the normalized Jacquet module of $\pi$ along $P$. Precisely, $V_{N}$ is the quotient of $V$ by the $P$-stable subspace $V(N)=\operatorname{span}\{\pi(n) v-v: n \in$ $N, v \in V\}$, and the action of $P$ on $V_{N}$ is normalized by $\delta_{P}^{-1 / 2}$. The unipotent radical of $N$ acts trivially on $\left(\pi_{N}, V_{N}\right)$ and we will regard $\left(\pi_{N}, V_{N}\right)$ as a representation of the Levi factor $M \cong P / N$ of $P$.

The Geometric Lemma (Lemma 2.1) is a fundamental tool in the study of induced representations. Let $P=M N$ and $Q=L U$ be two parabolic subgroups of $G$ with Levi factors $M$ and $L$, and unipotent radicals $N$ and $U$ respectively. Following [27], let

$$
S(M, L)=\left\{y \in G: M \cap{ }^{y} L \text { contains a maximal } F \text {-split torus of } G\right\} .
$$

There is a canonical bijection between the double-coset space $P \backslash G / Q$ and the set $M \backslash S(M, L) / L$. Let $y \in S(M, L)$. The subgroup $M \cap^{y} Q$ is a parabolic subgroup of $M$ and $P \cap{ }^{y} L$ is a parabolic subgroup of ${ }^{y} L$. The unipotent radical of $M \cap{ }^{y} Q$ is $M \cap{ }^{y} U$ and the unipotent radical of $P \cap{ }^{y} L$ is $N \cap{ }^{y} L$; 
moreover, $M \cap{ }^{y} L$ is a Levi subgroup of both $M \cap{ }^{y} Q$ and $P \cap{ }^{y} L$. Given a representation $\rho$ of $L$, we obtain a representation ${ }^{y} \rho=\rho \circ \operatorname{Int} y^{-1}$ of ${ }^{y} L$.

Lemma 2.1 (The Geometric Lemma, [3, Lemma 2.12]). Let $\rho$ be a smooth representation of $L$. There is a filtration of the space of the representation $\left(\iota_{Q}^{G} \rho\right)_{N}$ such that the associated graded object is isomorphic to the direct sum

$$
\bigoplus_{y \in M \backslash S(M, L) / L} \iota_{M \cap^{y} Q}^{M}\left(\left({ }^{y} \rho\right)_{N \cap^{y} L}\right) .
$$

Remark 2.2. We will write $\mathcal{F}_{N}^{y}(\rho)$ to denote the smooth representation $\iota_{M \cap^{y} Q}^{M}\left(\left({ }^{y} \rho\right)_{N \cap^{y} L}\right)$ of $M$.

Let $\Phi$ be the root system of $G$ (relative to a choice of maximal $F$-split torus $A_{0}$ ). Fix a base $\Delta_{0}$ for $\Phi$ and let $W_{0}$ be the Weyl group of $G$ (with respect to $A_{0}$ ). The choice of $\Delta_{0}$ determines a system $\Phi^{+}$of positive roots. Given a subset $\Theta$ of $\Delta_{0}$, we may associate a standard parabolic subgroup $P_{\Theta}=M_{\Theta} N_{\Theta}$, with Levi factor $M_{\Theta}$ and unipotent radical $N_{\Theta}$, in the usual way. The following lemma gives a good choice of Weyl group representatives to use when applying Lemma 2.1 for standard parabolic subgroups.

Lemma 2.3 ([6, Proposition 1.3.1]). Let $\Theta$ and $\Omega$ be subsets of $\Delta_{0}$. The set

$$
\left[W_{\Theta} \backslash W_{0} / W_{\Omega}\right]=\left\{w \in W_{0}: w \Omega, w^{-1} \Theta \subset \Phi^{+}\right\}
$$

provides a choice of Weyl group representatives for the double-coset space $P_{\Theta} \backslash G / P_{\Omega}$.

We will always use the choice of "nice" representatives $\left[W_{\Theta} \backslash W_{0} / W_{\Omega}\right] \subset$ $S\left(M_{\Theta}, M_{\Omega}\right)$ for the double-coset space $P_{\Theta} \backslash G / P_{\Omega} \simeq M_{\Theta} \backslash S\left(M_{\Theta}, M_{\Omega}\right) / M_{\Omega}$.

2.2. Distinguished (induced) representations. Let $\pi$ be a smooth representation of $G$. We also let $\pi$ denote its restriction to $H$. Let $\chi$ be a quasi-character of $H$.

Definition 2.4. The representation $\pi$ is $(H, \chi)$-distinguished if the space $\operatorname{Hom}_{H}(\pi, \chi)$ is nonzero.

If $\pi$ is $(H, 1)$-distinguished, where 1 is the trivial character of $H$, then we will simply call $\pi H$-distinguished. The elements of $\operatorname{Hom}_{H}(\pi, 1)$ are $H$-invariant linear forms on the space of $\pi$.

2.2.1. Relative matrix coefficients. Let $(\pi, V)$ be a smooth $H$-distinguished representation of $G$. Let $\lambda \in \operatorname{Hom}_{H}(\pi, 1)$ be a nonzero $H$-invariant linear form on $V$ and let $v$ be a nonzero vector in $V$. In analogy with the usual matrix coefficients, define a complex-valued function $\varphi_{\lambda, v}$ on $G$ by $\varphi_{\lambda, v}(g)=\langle\lambda, \pi(g) v\rangle$. We refer to the functions $\varphi_{\lambda, v}$ as $\lambda$-relative matrix coefficients. When $\lambda$ is understood, we will drop it from the terminology. The representation $\pi$ is smooth; therefore, the relative matrix coefficients 
$\varphi_{\lambda, v}$ lie in $C^{\infty}(G)$, for every $v \in V$. In addition, since $\lambda$ is $H$-invariant, the functions $\varphi_{\lambda, v}$ descend to well-defined functions on the quotient $H \backslash G$.

Let $\omega$ be a unitary character of $Z_{G}$ and further suppose that $\pi$ is an $\omega$-representation. Since the central character $\omega$ is unitary, the function $Z_{G} H \cdot g \mapsto\left|\varphi_{\lambda, v}(g)\right|$ is well defined on $Z_{G} H \backslash G$. The centre $Z_{G}$ of $G$ is unimodular since it is abelian. The fixed point subgroup $H$ is also reductive (cf. [7, Theorem 1.8]) and thus unimodular. It follows that there exists a $G$-invariant measure on the quotient $Z_{G} H \backslash G$ by [26, Proposition 12.8].

Definition 2.5. The representation $(\pi, V)$ is said to be

(1) $(H, \lambda)$-relatively square integrable if and only if all of the $\lambda$-relative matrix coefficients are square integrable modulo $Z_{G} H$.

(2) $H$-relatively square integrable if and only if $\pi$ is $(H, \lambda)$-relatively square integrable for every $\lambda \in \operatorname{Hom}_{H}(\pi, 1)$.

When $H$ is understood, we drop it from the terminology and speak of relatively square integrable representations. If $(\pi, V)$ is $H$-distinguished and $(H, \lambda)$-relatively square integrable, then the morphism that sends $v \in V$ to the relative matrix coefficient $\varphi_{\lambda, v}$ is an intertwining operator from $\pi$ to the right regular representation of $G$ on $L^{2}\left(Z_{G} H \backslash G, \omega\right)$, where $L^{2}\left(Z_{G} H \backslash G, \omega\right)$ is the space of functions on $H \backslash G$, square integrable modulo $Z_{G}$, that are $Z_{G}$-eigenfunctions with eigencharacter $\omega$.

Definition 2.6. If $(\pi, V)$ is an irreducible subrepresentation of $L^{2}\left(Z_{G} H \backslash G\right)$, then we say that $(\pi, V)$ occurs in the discrete spectrum of $H \backslash G$. In this case, we say that $(\pi, V)$ is a relative discrete series (RDS) representation.

The main goal of the present paper is to construct RDS representations for $\mathbf{U}_{E / F}(F) \backslash \mathbf{G L}_{2 n}(E)$, when $\mathbf{U}_{E / F}$ is quasi-split unitary group over $F$.

2.2.2. Invariant forms on induced representations. Lemma 2.7 is well known and follows from an explicit version of Frobenius Reciprocity due to Bernstein and Zelevinsky [2, Proposition 2.29]. Let $Q=L U$ be a $\theta$-stable parabolic subgroup with $\theta$-stable Levi factor $L$ and unipotent radical $U$. Note that the identity component of $Q^{\theta}=L^{\theta} U^{\theta}$ is a parabolic subgroup of the identity component $H^{\circ}$ of $H$, with the expected Levi decomposition ( $c f$. [13], [10. Lemma 3.1]). Let $\mu$ be a positive quasi-invariant measure on the quotient $Q^{\theta} \backslash H$ [2, Theorem 1.21].

Lemma 2.7. Let $\rho$ be a smooth representation of $L$ and let $\pi=\iota_{Q}^{G} \rho$. The map $\lambda \mapsto \lambda^{G}$ is an injection of $\operatorname{Hom}_{L^{\theta}}\left(\delta_{Q}^{1 / 2} \rho, \delta_{Q^{\theta}}\right)$ into $\operatorname{Hom}_{H}(\pi, 1)$, where for any function $\phi$ in the space of $\pi, \lambda^{G}$ is given explicitly by

$$
\left\langle\lambda^{G}, \phi\right\rangle=\int_{Q^{\theta} \backslash H}\langle\lambda, \phi(h)\rangle d \mu(h) .
$$

Corollary 2.8. If $\delta_{Q}^{1 / 2}$ restricted to $L^{\theta}$ is equal to $\delta_{Q^{\theta}}$, then the map $\lambda \mapsto \lambda^{G}$ is an injection of $\operatorname{Hom}_{L^{\theta}}(\rho, 1)$ into $\operatorname{Hom}_{H}(\pi, 1)$. In particular, if $\rho$ is $L^{\theta}$ distinguished, then $\pi$ is $H$-distinguished. 
Proof. Observe that $\operatorname{Hom}_{L^{\theta}}\left(\delta_{Q}^{1 / 2} \rho, \delta_{Q^{\theta}}\right)=\operatorname{Hom}_{L^{\theta}}\left(\rho,\left.\delta_{Q}^{-1 / 2}\right|_{L^{\theta}} \delta_{Q^{\theta}}\right)$.

In fact, the $H$-invariant linear form on $\pi=\iota_{Q}^{G} \rho$ arises from the closed orbit in $Q \backslash G / H$ via the Mackey theory.

\section{Background on RDS: the Relative Casselman's Criterion}

3.1. Exponents (of induced representations). Let $(\pi, V)$ be a finitely generated admissible representation of $G$. Let $\chi$ be a quasi-character of the $F$-split component $A_{G}$ of the centre of $G$. For $n \in \mathbb{N}, n \geq 1$, define the subspace

$$
V_{\chi, n}=\left\{v \in V:(\pi(z)-\chi(z))^{n} v=0, \text { for all } z \in A_{G}\right\},
$$

and set

$$
V_{\chi}=\bigcup_{n=1}^{\infty} V_{\chi, n} .
$$

Each $V_{\chi, n}$ is a $G$-stable subspace of $V$ and $V_{\chi}$ is the generalized $\chi$-eigenspace in $V$ for the $A_{G}$-action on $V$. By [6, Proposition 2.1.9],

(1) $V$ is a direct sum $V=\bigoplus_{\chi} V_{\chi}$, where $\chi$ ranges over quasi-characters of $A_{G}$, and

(2) since $V$ is finitely generated, there are only finitely many $\chi$ such that $V_{\chi} \neq 0$. Moreover, there exists $n \in \mathbb{N}$ such that $V_{\chi}=V_{\chi, n}$, for each $\chi$.

Let $\mathscr{E}_{x p_{A_{G}}}(\pi)$ be the (finite) set of quasi-characters of $A_{G}$ such that $V_{\chi} \neq 0$. The quasi-characters that appear in $\mathscr{E} x p_{A_{G}}(\pi)$ are called the exponents of $\pi$. The second item above implies that $V$ has a finite filtration such that the quotients are $\chi$-representations, for $\chi \in \mathscr{E} x p_{A_{G}}(\pi)$.

Lemma 3.1. The characters $\chi$ of $A_{G}$ that appear in $E x p_{A_{G}}(\pi)$ are the central quasi-characters of the irreducible subquotients of $\pi$.

Let $(\pi, V)$ be a finitely generated admissible representation of $G$. Let $P=M N$ be a parabolic subgroup of $G$ with Levi factor $M$ and unipotent radical $N$. It is a theorem of Jacquet that $\left(\pi_{N}, V_{N}\right)$ is also finitely generated and admissible (cf. 66, Theorem 3.3.1]). Applying (1) and (2) to $\left(\pi_{N}, V_{N}\right)$, we obtain a direct sum decomposition

$$
V_{N}=\bigoplus_{\chi \in \in \operatorname{Ex}_{A_{M}}\left(\pi_{N}\right)}\left(V_{N}\right)_{\chi}
$$

where the set $\operatorname{Exp}_{A_{M}}\left(\pi_{N}\right)$ of quasi-characters of $A_{M}$, such that $\left(V_{N}\right)_{\chi} \neq 0$, is finite. The quasi-characters of $A_{M}$ appearing in $\varepsilon_{x p} p_{A_{M}}\left(\pi_{N}\right)$ are called the exponents of $\pi$ along $P$.

We are ultimately interested in the exponents of parabolically induced representations. For a proof of the following lemma, see [29, Lemma 4.15] 
Lemma 3.2. Let $P=M N$ be a parabolic subgroup of $G$, let $\left(\rho, V_{\rho}\right)$ be a finitely generated admissible representation of $M$ and let $\pi=\iota_{P}^{G} \rho$. The quasi-characters $\chi \in \mathcal{E x p}_{A_{G}}(\pi)$ are the restriction to $A_{G}$ of characters $\eta$ of $A_{M}$ appearing in $E_{x p_{A_{M}}}(\rho)$.

3.2. Invariant linear forms on Jacquet modules. Let $(\pi, V)$ be an admissible $H$-distinguished representation of $G$. Let $\lambda$ be a nonzero $H$ invariant linear form on $V$. Let $P$ be a $\theta$-split parabolic subgroup of $G$. Recall that a parabolic subgroup $P$ of $G$ is $\theta$-split if $\theta(P)$ is opposite to $P$ (cf. Section 4.2). Let $N$ be the unipotent radical of $P$, and let $M=P \cap \theta(P)$ be a $\theta$-stable Levi factor of $P$. Independently, Kato-Takano and Lagier define an $M^{\theta}$-invariant linear form $\lambda_{N}$ on the Jacquet module $\left(\pi_{N}, V_{N}\right)$. The construction of $\lambda_{N}$ relies on Casselman's Canonical Lifting [6, Proposition 4.1.4]. We next record [20, Proposition 5.6], and we refer the interested reader to [20, 22] for the details of the construction of $\lambda_{N}$.

Proposition 3.3 (Kato-Takano, Lagier). Let $(\pi, V)$ be an admissible $H$ distinguished representation of $G$. Let $\lambda \in \operatorname{Hom}_{H}(\pi, 1)$ be nonzero and let $P$ be a $\theta$-split parabolic subgroup of $G$ with unipotent radical $N$ and $\theta$-stable Levi component $M=P \cap \theta(P)$.

(1) The linear functional $\lambda_{N}: V_{N} \rightarrow \mathbb{C}$ is $M^{\theta}$-invariant.

(2) The mapping $\operatorname{Hom}_{H}(\pi, 1) \rightarrow \operatorname{Hom}_{M^{\theta}}\left(\pi_{N}, 1\right)$, sending $\lambda$ to $\lambda_{N}$, is linear.

3.3. The Relative Casselman's Criterion. Let $(\pi, V)$ be a finitely generated admissible $H$-distinguished representation of $G$. Fix a nonzero $H$ invariant form $\lambda$ on $V$. For any closed subgroup $Z$ of the centre of $G$, Kato and Takano 21] define

$$
\mathcal{E x p}_{Z}(\pi, \lambda)=\left\{\chi \in \mathcal{E} x p_{Z}(\pi):\left.\lambda\right|_{V_{\chi}} \neq 0\right\},
$$

and refer to the set $\varepsilon x p_{Z}(\pi, \lambda)$ as exponents of $\pi$ relative to $\lambda$.

The following appears as [21, Theorem 4.7], see Section 4.1 for the definition of the set $S_{M}^{-} \backslash S_{G} S_{M}^{1}$.

Theorem 3.4 (The Relative Casselman's Criterion, Kato-Takano). Let $\omega$ be a unitary character of $Z_{G}$. Let $(\pi, V)$ be a finitely generated admissible $H$ distinguished $\omega$-representation of $G$. Fix a nonzero $H$-invariant linear form $\lambda$ on $V$. The representation $(\pi, V)$ is $(H, \lambda)$-relatively square integrable if and only if the condition

$$
|\chi(s)|<1 \quad \text { for all } \chi \in E_{x} p_{S_{M}}\left(\pi_{N}, \lambda_{N}\right) \text { and all } s \in S_{M}^{-} \backslash S_{G} S_{M}^{1}
$$

is satisfied for every proper $\theta$-split parabolic subgroup $P=M N$ of $G$.

It is an immediate corollary of Theorem 3.4 that: if $(\pi, V)$ is an $H$ distinguished discrete series representation of $G$, then $\pi$ is $H$-relatively square integrable. For a proof of the following, see [29, Proposition 4.22]. 
Proposition 3.5. Let $(\pi, V)$ be a finitely generated admissible represen-

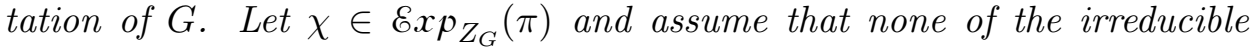
subquotients of $(\pi, V)$ with central character $\chi$ are $H$-distinguished. Then for any $\lambda \in \operatorname{Hom}_{H}(\pi, 1)$, the restriction of $\lambda$ to $V_{\chi}$ is equal to zero, i.e., $\left.\lambda\right|_{V_{\chi}} \equiv 0$.

\section{4. $p$-ADIC SYMMETRIC SPACES AND PARABOLIC SUBGROUPS}

In this section, we discuss the tori, root systems, and parabolic subgroups relevant to our study of $H \backslash G$. We briefly review some general notions before turning our attention to the case of $\mathbf{U}_{E / F}(F) \backslash \mathbf{G L}_{2 n}(E)$ in Section 4.3.

4.1. $(\theta, F)$-split tori and the relative root system. We say that an element $g \in G$ is $\theta$-split if $\theta(g)=g^{-1}$. Recall that an $F$-torus $S$ contained in $G$ is $(\theta, F)$-split if $S$ is $F$-split and every element of $S$ is $\theta$-split. Let $S_{0}$ be a maximal $(\theta, F)$-split torus of $G$. Fix a $\theta$-stable maximal $F$-split torus $A_{0}$ of $G$ that contains $S_{0}$ [13, Lemma 4.5(iii)]. Let $\Phi_{0}=\Phi\left(G, A_{0}\right)$ be the root system of $G$ with respect to $A_{0}$, and let $W_{0}$ be the associated Weyl group.

Let $M$ be any Levi subgroup of $G$. Let $A_{M}$ be the $F$-split component of the centre of $M$. The $(\theta, F)$-split component of $M$ is the largest $(\theta, F)$-split torus $S_{M}$ contained in $Z_{M}$. The torus $S_{M}$ is the connected component of the subgroup of $\theta$-split elements in $A_{M}$. Explicitly $\left.\right|^{3}$

$$
S_{M}=\left(\left\{x \in A_{M}: \theta(x)=x^{-1}\right\}\right)^{\circ} .
$$

There is an action of $\theta$ on the $F$-rational characters $X^{*}\left(A_{0}\right)$ of $A_{0}$. Indeed, since $A_{0}$ is $\theta$-stable, for $\chi \in X^{*}\left(A_{0}\right)$, the character

$$
(\theta \chi)(a)=\chi(\theta(a))
$$

is well defined for all $a \in A_{0}$. In addition, $\Phi_{0} \subset X^{*}\left(A_{0}\right)$ is stable under the action of $\theta$. Let $\Phi_{0}^{\theta}$ be the set of $\theta$-fixed roots. Recall that a choice $\Delta_{0}$ of base for $\Phi_{0}$ determines a system $\Phi_{0}^{+}$of positive roots.

Definition 4.1. A base $\Delta_{0}$ of $\Phi_{0}$ is called a $\theta$-base if for every $\alpha \in \Phi_{0}^{+}$, such that $\alpha \neq \theta(\alpha)$, we have that $\theta(\alpha) \in \Phi_{0}^{-}$.

Let $\Delta_{0}$ be a $\theta$-base of $\Phi_{0}$ (existence of a $\theta$-base is proved in [14]). Let $p: X^{*}\left(A_{0}\right) \rightarrow X^{*}\left(S_{0}\right)$ be the morphism defined by restricting the $F$-rational characters of $A_{0}$ to the subtorus $S_{0}$. The map $p$ is surjective and the kernel of $p$ is the submodule $X^{*}\left(A_{0}\right)^{\theta}$ consisting of $\theta$-fixed $F$-rational characters. The restricted root system of $H \backslash G$ (relative to our choice of $\left(A_{0}, S_{0}, \Delta_{0}\right)$ ) is defined to be

$$
\bar{\Phi}_{0}=p\left(\Phi_{0}\right) \backslash\{0\}=p\left(\Phi_{0} \backslash \Phi_{0}^{\theta}\right) .
$$

\footnotetext{
${ }^{3}$ Here $(\cdot)^{\circ}$ indicates the Zariski-connected component of the identity.
} 
The set $\bar{\Phi}_{0}$ coincides with the set $\Phi\left(G, S_{0}\right)$ of roots in $G$ with respect to $S_{0}$. The set $\bar{\Phi}_{0}$ is a root system by [13, Proposition 5.9]; however, $\bar{\Phi}_{0}$ is not necessarily reduced. The set

$$
\bar{\Delta}_{0}=p\left(\Delta_{0}\right) \backslash\{0\}=p\left(\Delta_{0} \backslash \Delta_{0}^{\theta}\right)
$$

forms a base for $\bar{\Phi}_{0}$. The linear independence of $\bar{\Delta}_{0}$ follows from the fact that $\Delta_{0}$ is a $\theta$-base and that $\operatorname{ker} p=X^{*}\left(A_{0}\right)^{\theta}$ consists of $\theta$-fixed characters.

Given a subset $\bar{\Theta} \subset \bar{\Delta}_{0}$, define the subset

$$
[\bar{\Theta}]=p^{-1}(\bar{\Theta}) \cup \Delta_{0}^{\theta}
$$

of $\Delta_{0}$. Subsets of $\Delta_{0}$ of the form $[\bar{\Theta}]$, where $\bar{\Theta} \subset \bar{\Delta}_{0}$, are called $\theta$-split. The maximal $\theta$-split subsets of $\Delta_{0}$ are of the form $\left[\bar{\Delta}_{0} \backslash\{\bar{\alpha}\}\right]$, where $\bar{\alpha} \in \bar{\Delta}_{0}$.

4.2. $\theta$-split parabolic subgroups and $\theta$-elliptic Levi factors. As above, let $\Delta_{0}$ be a $\theta$-base of $\Phi_{0}$. To any subset $\Theta$ of $\Delta_{0}$, we may associate a $\Delta_{0}$-standard parabolic subgroup $P_{\Theta}$ of $G$ with unipotent radical $N_{\Theta}$ and standard Levi factor $M_{\Theta}=C_{G}\left(A_{\Theta}\right)$, where $A_{\Theta}$ is the $F$-split torus

$$
A_{\Theta}=\left(\bigcap_{\alpha \in \Theta} \operatorname{ker} \alpha\right)^{\circ} \text {. }
$$

Let $\Phi_{\Theta}$ be the subsystem of $\Phi_{0}$ generated by the simple roots $\Theta$. Let $\Phi_{\Theta}^{+}$be the system of $\Theta$-positive roots. The unipotent radical $N_{\Theta}$ of $P_{\Theta}$ is generated by the root groups $N_{\alpha}$, where $\alpha \in \Phi_{0}^{+} \backslash \Phi_{\Theta}^{+}$. The torus $A_{\Theta}$ is the $F$-split component of the centre of $M_{\Theta}$ and $\Phi_{\Theta}$ is the root system of $A_{0}$ in $M_{\Theta}$.

Definition 4.2. A parabolic subgroup $P$ of $G$ is $\theta$-split if $\theta(P)$ is opposite to $P$.

If $P$ is a $\theta$-split parabolic subgroup, then $M=P \cap \theta(P)$ is a $\theta$-stable Levi subgroup of both $P$ and the opposite parabolic $P^{\mathrm{op}}=\theta(P)$. If $\Theta \subset \Delta_{0}$ is $\theta$ split, then the $\Delta_{0}$-standard parabolic subgroup $P_{\Theta}=M_{\Theta} N_{\Theta}$ is $\theta$-split. Any $\Delta_{0}$-standard $\theta$-split parabolic subgroup arises this way [20, Lemma 2.5(1)]. Following [21, §1.5], the $(\theta, F)$-split component of $M_{\Theta}$ is equal to

$$
S_{\Theta}=\left(\left\{s \in A_{\Theta}: \theta(s)=s^{-1}\right\}\right)^{\circ}=\left(\bigcap_{\bar{\alpha} \in p(\Theta)} \operatorname{ker}\left(\bar{\alpha}: S_{0} \rightarrow F^{\times}\right)\right)^{\circ} .
$$

For any $0<\epsilon \leq 1$, define

$$
S_{\Theta}^{-}(\epsilon)=\left\{s \in S_{\Theta}:|\alpha(s)|_{F} \leq \epsilon, \text { for all } \alpha \in \Delta_{0} \backslash \Theta\right\} .
$$

Let $S_{\Theta}^{-}$denote $S_{\Theta}^{-}(1)$. The set $S_{\Theta}^{-}$is referred to as the dominant part of $S_{\Theta}$.

By [12, Theorem 2.9], the subset $\Delta_{0}^{\theta}$ of $\theta$-fixed roots in $\Delta_{0}$ determines the $\left(\Delta_{0}\right.$-standard $)$ minimal $\theta$-split parabolic subgroup $P_{0}=P_{\Delta_{0}^{\theta}}$. By [13, Proposition 4.7(iv)], the minimal $\theta$-split parabolic subgroup $P_{0}$ has standard $\theta$-stable Levi $M_{0}=C_{G}\left(S_{0}\right)$. Let $N_{0}$ be the unipotent radical of $P_{0}$. We have that $P_{0}=M_{0} N_{0}$. 
Lemma 4.3 ([20, Lemma 2.5]). Let $S_{0} \subset A_{0}, \Delta_{0}$, and $P_{0}=M_{0} N_{0}$ be as above.

(1) Any $\theta$-split parabolic subgroup $P$ of $G$ is conjugate to a $\Delta_{0}$-standard $\theta$-split parabolic subgroup by an element $g \in\left(\mathbf{H M}_{0}\right)(F)$.

(2) If the group of $F$-points of the product $\left(\mathbf{H M}_{0}\right)(F)$ is equal to $H M_{0}$, then any $\theta$-split parabolic subgroup of $G$ is $H$-conjugate to a $\Delta_{0^{-}}$ standard $\theta$-split parabolic subgroup.

Let $P=M N$ be a $\theta$-split parabolic subgroup. Pick $g \in\left(\mathbf{H M}_{0}\right)(F)$ such that $P=g P_{\Theta} g^{-1}$ for some $\theta$-split subset $\Theta \subset \Delta_{0}$. Since $g \in\left(\mathbf{H M}_{0}\right)(F)$ we have that $g^{-1} \theta(g) \in \mathbf{M}_{0}(F)$, and we have $S_{M}=g S_{\Theta} g^{-1}$. For a given $\epsilon>0$, one may extend the definition of $S_{\Theta}^{-}$in (4.1) to the torus $S_{M}$. Set $S_{M}^{-}(\epsilon)=g S_{\Theta}^{-}(\epsilon) g^{-1}$ and define $S_{M}^{-}=S_{M}^{-}(1)$. Recall that we write $S_{M}^{1}$ to denote the $\mathcal{\Theta}_{F}$-points $S_{M}\left(\mathcal{\Theta}_{F}\right)$.

The next definition is made in analogy with the notion of an elliptic Levi subgroup. The following terminology is due to Murnaghan [25].

Definition 4.4. A $\theta$-stable Levi subgroup $L$ of $G$ is $\theta$-elliptic if and only if $L$ is not contained in any proper $\theta$-split parabolic subgroup of $G$.

The next lemma follows immediately from Definition 4.4.

Lemma 4.5. If a $\theta$-stable Levi subgroup $L$ of $G$ contains a $\theta$-elliptic Levi subgroup, then $L$ is $\theta$-elliptic.

The next proposition appears in [25, Proof of Proposition 8.4].

Proposition 4.6. Let $Q$ be a parabolic subgroup of $G$. If $Q$ admits a $\theta$ elliptic Levi factor $L$, then $Q$ is $\theta$-stable.

Proof. By definition, $L$ is $\theta$-stable. One can show that for any root $\alpha$ of $A_{L}$ in $G$ we have $\theta(\alpha)=\alpha$. It follows that the unipotent radical of $Q$ is also $\theta$-stable.

4.3. Structure of $\mathbf{U}_{E / F}(F) \backslash \mathbf{G L}_{2 n}(E)$. Let $\mathbf{G}=R_{E / F} \mathbf{G} \mathbf{L}_{n}$ be the restriction of scalars from $E$ to $F$ of $\mathbf{G L}_{n}$. We will restrict to the case that $n$ is even in Section 5 (onward). We identify the group $G=\mathbf{G}(F)$ with the set $\mathbf{G L}_{n}(E)$, of $E$-points of $\mathbf{G} \mathbf{L}_{n}$. The non-trivial element $\sigma$ of the Galois group $\operatorname{Gal}(E / F)$ gives rise to an $F$-involution of $\mathbf{G}$ given by entry-wise Galois conjugation on $\mathbf{G L}_{n}(E)$. We denote the Galois involution of $\mathbf{G}$ by $\sigma$. Explicitly,

$$
\sigma(g)=\left(\sigma\left(g_{i j}\right)\right), \quad \text { where } g=\left(g_{i j}\right) \in G .
$$

Following [8], let $\mathbf{X}$ denote the $F$-variety of Hermitian matrices in $\mathbf{G}$,

$$
\mathbf{X}=\left\{x \in \mathbf{G}:{ }^{t} \sigma(x)=x\right\} .
$$

Here ${ }^{t} g$ denotes the transpose of $g \in \mathbf{G}$. There is a right action of $\mathbf{G}$ on $\mathbf{X}$ given by $x \cdot g={ }^{t} \sigma(g) x g$, where $x \in \mathbf{X}$ and $g \in \mathbf{G}$. Write $X=$ $\mathbf{X}(F)$ for the $F$ points of $\mathbf{X}$. There is a finite set $X / G$ of $G$-orbits in $X$ 
indexed by $F^{\times} / N_{E / F}\left(E^{\times}\right)$[8]. By Local Class Field Theory, $F^{\times} / N_{E / F}\left(E^{\times}\right)$ is isomorphic to $\operatorname{Gal}(E / F)$, and thus consists of two elements.

Given $x \in X$, define an $F$-involution $\theta_{x}$ of $G$ by

$$
\theta_{x}(g)=x^{-1 t} \sigma(g)^{-1} x,
$$

for all $g \in G$. Let $\mathbf{H}^{x}=\mathbf{G}^{\theta_{x}}$ be the subgroup of $\theta_{x}$-fixed elements. The group of $F$-points $H^{x}=\mathbf{H}^{x}(F)$ is a unitary group associated to $E / F$ and $x$.

Remark 4.7. In the literature, $\mathbf{U}_{E / F, x}$ is often used to denote the unitary group $\mathbf{H}^{x}$ associated to $E / F$ and $x$. We will use the $\mathbf{U}_{E / F, x}$ notation for unitary groups that appear as subgroups of Levi factors of $G$.

Definition 4.8. An involution $\theta_{1}$ of $G$ is $G$-equivalent to another involution $\theta_{2}$ if there exists $g \in G$ such that $\theta_{1}=\operatorname{Int} g^{-1} \circ \theta_{2} \circ \operatorname{Int} g$, where Int $g$ denotes the inner $F$-automorphism of $\mathbf{G}$ given by $\operatorname{Int} g(x)=g x g^{-1}$, for all $x \in \mathbf{G}$. We write $g \cdot \theta$ to denote the involution Int $g^{-1} \circ \theta \circ \operatorname{Int} g$.

Two involutions $\theta_{x_{1}}$ and $\theta_{x_{2}}$ are $G$-equivalent if and only if $x_{1}$ and $x_{2}$ lie in the same $G$-orbit in $X / G$. Indeed, if there exists $g \in G$ such that $y=x \cdot g={ }^{t} \sigma(g) x g$, then one can check that $\theta_{y}$ is equal to the involution $g \cdot \theta_{x}=\operatorname{Int} g^{-1} \circ \theta_{x} \circ \operatorname{Int} g$. Note that the $G$-action $\theta \mapsto g \cdot \theta$ on involutions is also a right-action. Since $X / G$ has order two, there are two $G$-equivalence classes of involutions of the form $\theta_{x}$. It is well known that when $n$ is odd, $\mathbf{H}^{x}$ is always quasi-split over $F$. When $n$ is even there are two isomorphism classes of unitary group associated to $E / F$, one of which is quasi-split.

We fix $\theta=\theta_{w_{\ell}}$, where $w_{\ell}$ is the permutation matrix in $G$ with unit antidiagonal, and write $\mathbf{H}=\mathbf{G}^{\theta}$. The group $\mathbf{H}=\mathbf{U}_{E / F, w_{\ell}}$ is quasi-split over $F$. Write $H=\mathbf{H}(F)$ for the group of $F$-points of $\mathbf{H}$.

Let $J_{r}$ be the $r \times r$ permutation matrix with unit anti-diagonal

$$
J_{r}=\left(\begin{array}{lll} 
& & 1 \\
& & \cdot \\
1 & &
\end{array}\right)
$$

and note that $w_{\ell}=J_{n}$. For any positive integer $r$, there exists $\gamma_{r} \in \mathbf{G L}_{r}(E)$ such that ${ }^{t} \sigma\left(\gamma_{r}\right) J_{r} \gamma_{r}$ lies in the diagonal $F$-split torus of $\mathbf{G L}_{r}(E)$. For instance if $r$ is even, we set

$$
\gamma_{r}=\left(\begin{array}{cccccc}
1 & & & & & 1 \\
& \ddots & & & . & \\
& & 1 & 1 & & \\
& & 1 & -1 & & \\
& . \cdot & & & \ddots & \\
1 & & & & & -1
\end{array}\right)
$$


and if $r$ is odd, we take

$$
\gamma_{r}=\left(\begin{array}{ccccccc}
1 & & & & & & 1 \\
& \ddots & & & & . & \\
& & 1 & 0 & 1 & & \\
& & 0 & 1 & 0 & & \\
& & 1 & 0 & -1 & & \\
& . & & & & \ddots & \\
1 & & & & & & -1
\end{array}\right) .
$$

Define $\gamma=\gamma_{n}$ and notice that

$$
{ }^{t} \sigma(\gamma) w_{\ell} \gamma=\operatorname{diag}(\underbrace{2, \ldots, 2}_{\left\lfloor\frac{n}{2}\right\rfloor}, \widehat{1}, \underbrace{-2, \ldots,-2}_{\left\lfloor\frac{n}{2}\right\rfloor}),
$$

lies in the diagonal $F$-split torus $A_{T}$ of $G$.

Let $\mathbf{T}$ be the maximal (non-split) diagonal $F$-torus of $\mathbf{G}$. The torus $\mathbf{T}$ is obtained by restriction of scalars of the diagonal torus of $\mathbf{G L}_{n}$. Let $T=\mathbf{T}(F)$, and identify $T$ with the diagonal matrices in $\mathbf{G L}_{n}(E)$. Let $A_{T}$ be the $F$-split component of $T$. Define $T_{0}={ }^{\gamma} T$, then the $F$-split component of $T_{0}$ is $A_{0}={ }^{\gamma} A_{T}$. The tori $T, A_{T}, T_{0}$ and $A_{0}$ are all $\theta$-stable. Observe that $A_{0}$ is a maximal $F$-split torus of $G$ that is $\theta$-split. In particular, $A_{0}$ is a maximal $(\theta, F)$-split torus of $G$. Indeed, we have that ${ }^{t} \sigma(\gamma) w_{\ell} \gamma$ lies in the abelian subgroup $A_{T}$; therefore, for any $\gamma t \gamma^{-1} \in A_{0}$, we have

$$
\begin{aligned}
\theta\left(\gamma t \gamma^{-1}\right) & =w_{\ell}^{-1 t} \sigma(\gamma)^{-1}\left({ }^{t} \sigma(t)^{-1}\right)^{t} \sigma(\gamma) w_{\ell} \\
& =\gamma\left({ }^{t} \sigma(\gamma) w_{\ell} \gamma\right)^{-1} t^{-1}\left({ }^{t} \sigma(\gamma) w_{\ell} \gamma\right) \gamma^{-1} \\
& =\left(\gamma t \gamma^{-1}\right)^{-1}
\end{aligned}
$$

where we've used that ${ }^{t} \sigma(t)^{-1}=t^{-1}$, for any $t \in A_{T}$.

Lemma 4.9. For any $x \in X$, the $\left(\theta_{x}, F\right)$-split component of $G$, which we denote by $S_{G, x}$, is equal to the $F$-split component $A_{G}$ of the centre of $G$.

Proof. Let $z \in A_{G}$. Since $z$ is a diagonal matrix with entries in $F^{\times}$, we have ${ }^{t} \sigma(z)=z$; moreover, since $z$ is central in $G$,

$$
\theta_{x}(z)=x^{-1 t} \sigma(z)^{-1} x=x^{-1} z^{-1} x=z^{-1},
$$

It follows that $S_{G, x}=\left(A_{G}\right)^{\circ}=A_{G}(c f$. Section 4.1).

Let $\Phi=\Phi\left(G, A_{T}\right)$ be the root system of $G$ with respect to $A_{T}$, with standard base $\Delta=\left\{\epsilon_{i}-\epsilon_{i+1}: 1 \leq i \leq n-1\right\}$. Let $\Phi_{0}=\Phi\left(G, A_{0}\right)$ be the root system of $G$ with respect to $A_{0}={ }^{\gamma} A_{T}$. Observe that $\Phi_{0}={ }^{\gamma} \Phi$. Set $\Delta_{0}=\gamma \Delta$. The set of positive roots of $\Phi_{0}$ with respect to $\Delta_{0}$ is denoted $\Phi_{0}^{+}$. We have that $\Phi_{0}^{+}={ }^{\gamma} \Phi^{+}$, where $\Phi^{+}$is the set of positive roots in $\Phi$ determined by $\Delta$. Our current aim is to use $\Phi_{0}$ to determine the (standard) $\theta$-split parabolic subgroups of $G$. First, we note the following.

Lemma 4.10. For any $\alpha \in \Phi_{0}$, we have $\theta(\alpha)=-\alpha$. 
Proof. Let $\alpha \in \Phi_{0}$. For any $a \in A_{0}$, we have that $\theta(a)=a^{-1}$; therefore,

$$
(\theta \alpha)(a)=\alpha(\theta(a))=\alpha\left(a^{-1}\right)=\alpha(a)^{-1}=(-\alpha)(a) .
$$

Since $a \in A_{0}$ was arbitrary, we have that $\theta(\alpha)=-\alpha$.

The following two corollaries of Lemma 4.10 follow immediately ( $c f$. Definition 4.1).

Corollary 4.11. The set $\Phi_{0}^{\theta}$ of $\theta$-fixed roots in $\Phi_{0}$ is empty.

Corollary 4.12. Any set of simple roots in $\Phi_{0}$ is a $\theta$-base for $\Phi_{0}$. In particular, $\Delta_{0}$ is a $\theta$-base.

Explicitly, $\Delta_{0}=\left\{\gamma\left(\epsilon_{i}-\epsilon_{i+1}\right): 1 \leq i \leq n-1\right\}$ and, by Corollary 4.12, the set of simple roots $\Delta_{0}$ is a $\theta$-base for $\Phi_{0}$. Since the maximal $F$-split torus $A_{0}$ is a maximal $(\theta, F)$-split torus, the restricted root system of $H \backslash G$ is just the root system $\Phi_{0}$ of $G$. The next proposition now follows immediately.

Proposition 4.13. Every parabolic subgroup of $G$ standard with respect to $\Delta_{0}$ is a $\theta$-split parabolic subgroup. Any such parabolic subgroup is the $\gamma$ conjugate of the usual block-upper triangular parabolic subgroups of $G$.

By Lemma 4.3(1), we have that any $\theta$-split parabolic subgroup of $G$ is $\left(\mathbf{H T}_{0}\right)(F)$-conjugate to a $\Delta_{0}$-standard $\theta$-split parabolic subgroup.

We now consider $\theta$-stable parabolic subgroups; in particular, we are concerned with determining which proper $\theta$-stable parabolic subgroups admit a $\theta$-elliptic Levi factor.

Definition 4.14. Let $(\underline{n})=\left(n_{1}, \ldots, n_{r}\right)$ be a partition of $n$, we say that $(\underline{n})$ is balanced if $n_{i}=n_{r+1-i}$, for $1 \leq i \leq r$.

Let $(\underline{n})=\left(n_{1}, \ldots, n_{r}\right)$ be a partition of $n$. The opposite partition to $(\underline{n})$ is $(\underline{n})^{\mathrm{op}}=\left(n_{r}, \ldots, n_{1}\right)$. This terminology reflects that the standard upper-triangular parabolic subgroup that is $\mathbf{G} \mathbf{L}_{n}$-conjugate to the (lowertriangular) opposite parabolic of $P_{(\underline{n})}$ is precisely $P_{(\underline{n})^{\text {op }}}$. Observe that $(\underline{n})$ is balanced if and only if $(\underline{n})^{\mathrm{op}}=(\underline{n})$.

Lemma 4.15. The $\theta$-stable block upper-triangular parabolic subgroups of $G$ correspond to balanced partitions of $n$. The only such parabolic that has a $\theta$-stable $\theta$-elliptic Levi subgroup is $P_{(n / 2, n / 2)}$, in the case that $n$ is even.

Proof. Let $(\underline{n})=\left(n_{1}, \ldots, n_{r}\right)$ be a partition of $n$. Let $A=A_{(\underline{n})}$ be the diagonal $F$-split torus corresponding to $(\underline{n})$. The parabolic subgroup $P=$ $P_{(\underline{n})}$ is $\theta$-stable if and only if its standard Levi subgroup $M=C_{G}(A)$ and unipotent radical $N$ are $\theta$-stable; moreover, $M$ is $\theta$-stable if and only if $A$ is $\theta$-stable. Observe that $A$ is $\theta$-stable if and only if $w_{\ell} \in N_{G}(A)$. Indeed, $\theta(a)=w_{\ell}^{-1 t} \sigma(a)^{-1} w_{\ell}$ and $A$ is stable under the involution $a \mapsto{ }^{t} \sigma(a)^{-1}=$ $a^{-1}$. It is immediate that $\theta(A)=A_{(\underline{n})^{\text {op }}}$; moreover, $A$ is $\theta$-stable if and only if $(\underline{n})^{\mathrm{op}}=(\underline{n})$ if and only if $(\underline{n})$ is balanced. Therefore, it suffices to show that if $N$ is $\theta$-stable if and only if $(\underline{n})$ is balanced. First note that $N$ is 
stable under the map $n \mapsto \sigma(n)^{-1}$, on the other hand, the transpose map sends $N$ to the opposite unipotent radical $N^{\mathrm{op}}$. In particular, $N$ is $\theta$-stable if and only if $N=w_{\ell}^{-1} N^{\mathrm{op}} w_{\ell}$. A simple matrix computation shows that this occurs if and only if $(\underline{n})^{\mathrm{op}}=(\underline{n})$, i.e., $(\underline{n})$ is balanced.

Let $(\underline{n})=\left(n_{1}, \ldots, n_{\lfloor r / 2\rfloor}, \widehat{n_{\bullet}}, n_{\lfloor r / 2\rfloor}, \ldots, n_{1}\right)$ be a balanced partition of $n$. Now, we show that $M$ is $\theta$-elliptic if and only if $(\underline{n})=(n / 2, n / 2)$ by applying [29, Lemma 3.8], which states that a $\theta$-stable Levi subgroup $M$ is $\theta$-elliptic if and only if $S_{M}=S_{G}$. An element $a=\operatorname{diag}\left(a_{1}, \ldots, a_{n}\right)$ of $A_{T}$ is $\theta$-split if and only if $a$ centralizes $w_{\ell}$. Indeed, since $A_{T}$ is pointwise fixed by taking the transpose-Galois conjugates, we have that

$$
\theta(a)=w_{\ell}^{-1} \operatorname{diag}\left(a_{1}^{-1}, \ldots, a_{n}^{-1}\right) w_{\ell}=\operatorname{diag}\left(a_{n}^{-1}, \ldots, a_{1}^{-1}\right),
$$

which is equal to $a^{-1}$ if and only if $a_{i}=a_{n+1-i}$, for all $1 \leq i \leq n$. It follows that $a^{-1}=\theta(a)$ if and only if $a \in C_{A_{T}}\left(w_{\ell}\right)$, where

$C_{A_{T}}\left(w_{\ell}\right)=\left\{\operatorname{diag}\left(a_{1}, \ldots, a_{\lfloor n / 2\rfloor}, \widehat{a}_{\bullet}, a_{\lfloor n / 2\rfloor}, \ldots, a_{1}\right): a_{i} \in F^{\times}, 1 \leq i \leq\lfloor n / 2\rfloor\right\}$.

The $(\theta, F)$-split component $S_{M}$ of $M$ is thus equal to the identity component of $A \cap C_{A_{T}}\left(w_{\ell}\right)$. We have that $A \cap C_{A_{T}}\left(w_{\ell}\right)$ is equal to the $F$-split torus

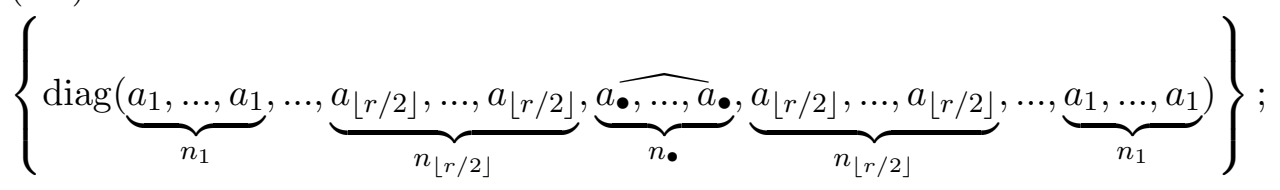

in particular, $S_{M}=A \cap C_{A_{T}}\left(w_{\ell}\right)$. By Lemma 4.9, we have $S_{G}=A_{G}$ and observe that $S_{M}$ is equal to $A_{G}$ if and only if $r=2$, that is, $n$ is even and $(\underline{n})=(n / 2, n / 2)$, as claimed.

Remark 4.16. When $n$ is even, we set $L=M_{(n / 2, n / 2)}$ and reiterate that $L$ is the only proper block-diagonal $\theta$-elliptic Levi subgroup of $G$.

Corollary 4.17. The minimal parabolic (Borel) subgroup $Q_{0}$ of $G$ consisting of the upper-triangular matrices is a $\theta$-stable minimal parabolic of $G$. In particular, $\mathbf{Q}_{0}=R_{E / F} \mathbf{B}$, where $\mathbf{B}$ is the upper-triangular Borel subgroup of $\mathbf{G L}_{n}$.

Proof. The partition $(1, \ldots, 1)$ is balanced; apply Lemma 4.15 .

Corollary 4.18. The F-subgroup $Q_{0} \cap H$ of $H$, consisting of the uppertriangular elements of $H$, is a Borel subgroup of $H$.

Proof. See, for instance, [10, Lemma 3.1].

Lemma 4.19. There are no proper $\theta$-elliptic Levi subgroups of $G$ that con$\operatorname{tain} A_{0}$.

Proof. The maximal $F$-split torus $A_{0}$ of $G$ is $(\theta, F)$-split. The lemma follows from [29, Lemma 3.8]. 
Recall that a parabolic subgroup $P$ of $G$ is called $A_{T}$-semi-standard if $P$ contains $A_{T}$. If $P$ is an $A_{T}$-semi-standard parabolic subgroup, then there is a unique Levi factor $M$ of $P$ that contains $A_{T}$. We refer to $M$ as the $A_{T}$-semi-standard Levi factor of $P$.

Lemma 4.20. Let $n \geq 2$ be an integer.

(1) If $n$ is odd, then there are no proper $\theta$-elliptic $A_{T}$-semi-standard Levi subgroups of $G=\mathbf{G L}_{n}(E)$.

(2) If $n$ is even, then $L=M_{(n / 2, n / 2)}$ is the only maximal proper $\theta$-elliptic $A_{T}$-semi-standard Levi subgroup of $G=\mathbf{G L}_{n}(E)$, up to conjugacy by Weyl group elements $w \in W=W\left(G, A_{T}\right)$, such that $w^{-1} w_{\ell} w \in$ $N_{G}(L) \backslash L$.

Proof. We give a sketch of the proof. We identify the Weyl group $W=$ $W\left(G, A_{T}\right)$ of $A_{T}$ in $G$ with the subgroup of permutation matrices in $G$. By Lemma 4.15, if $n$ even, then $L=M_{(n / 2, n / 2)}$ is $\theta$-elliptic.

First, let $P=M N$ be a $\theta$-stable maximal proper $A_{T}$-semi-standard parabolic subgroup of $G$ with $\theta$-stable $A_{T}$-semi-standard Levi subgroup $M$. It is well known that $P$ is $W$-conjugate to a unique standard (block upper-triangular) maximal parabolic subgroup of $G$. In particular, $M=$ $w M_{\left(n_{1}, n_{2}\right)} w^{-1}$ for some partition $\left(n_{1}, n_{2}\right)$ of $n$ and some $w \in W$. Moreover, $M$ is $\theta$-stable if and only if its $F$-split component $A_{M}=w A_{\left(n_{1}, n_{2}\right)} w^{-1}$ is contained in a torus $A_{(\underline{n})}$, for some balanced partition $(\underline{n})$ of $n$. Assume that $M$ is $\theta$-stable and let $(\underline{n})$ be the coarsest partition such that $w A_{\left(n_{1}, n_{2}\right)} w^{-1}$ is contained in $A_{(\underline{n})}$. One may regard the $F$-split component $A_{M}=w A_{\left(n_{1}, n_{2}\right)} w^{-1}$ of $M$ as being obtained by a two-colouring of $(\underline{n})$. That is, regard $\operatorname{diag}(\underbrace{a, \ldots, a}_{n_{1}}, \underbrace{b, \ldots, b}_{n_{2}}) \rightarrow A_{(\underline{n})}$ as a two-colouring of the partition $(\underline{n})$. One may readily verify that:

(1) If $n$ is odd, then, by considering 4.5), we see that $w A_{\left(n_{1}, n_{2}\right)} w^{-1}$ contains at least a rank-one $F$-split torus, non-central in $G$, consisting of $\theta$-split elements. (Indeed, since $n$ is odd, the central segment in (4.5) must appear.) In particular, $M$ cannot be $\theta$-elliptic by [29, Lemma 3.4]. Moreover, by Lemma 4.5, $M$ cannot contain a $\theta$-elliptic Levi subgroup of $G$. It follows that there are no $A_{T}$-semi-standard $\theta$-elliptic Levi subgroups when $n$ is odd.

(2) Suppose that $n$ is even. In light of (4.5), we see that $M$ is $\theta$-elliptic if and only if $(\underline{n})$ is a refinement of the partition $(n / 2, n / 2)$ of $n$. In particular, it readily follows that $n_{1}=n_{2}=n / 2$ and $M$ is conjugate to $L$.

Observe that $\theta(w)=w_{\ell}^{-1} w w_{\ell}$, for any $w \in W$. It is straightforward to check that $M=w L w^{-1}$ is $\theta$-stable if and only if $w^{-1} w_{\ell} w \in N_{G}(L)$. It can be verified that a $\theta$-stable conjugate $M=w L w^{-1}$ of $L$ is $\theta$-elliptic if and only if $w^{-1} w_{\ell} w \notin C_{G}\left(A_{L}\right)=L$. Thus $L$ is the only maximal $A_{T}$-semi-standard $\theta$-elliptic Levi subgroup of $G$ (up to conjugacy). 
Note. Observe that $L=M_{(n / 2, n / 2)}$ does not contain any proper $\theta$-elliptic Levi subgroups. We argue by contradiction. Suppose that $L^{\prime} \subsetneq L$ is a $\theta$-elliptic Levi subgroup of $G$. Notice that $L^{\prime}$ is also $\theta$-elliptic in $L$. Since $L^{\prime}$ is proper in $L$, it follows from Lemma 4.5 that $L^{\prime}$ is contained in a $\theta$ stable maximal proper Levi subgroup $L^{\prime \prime}$ of $L$. Without loss of generality, $L^{\prime \prime} \cong M_{\left(k_{1}, k_{2}\right)} \times \mathbf{G L}_{n / 2}(F)$. However, considering the action of $\theta$ on $L$ described in 4.8), we observe that no such Levi subgroup $L^{\prime \prime}$ can be $\theta$ stable.

Lemma 4.20 does not give a complete characterization of the $\theta$-elliptic Levi subgroups of $G$. The following lemma takes us closer to the desired result; in particular, up to $H$-conjugacy (and choice of standard parabolic) we have all of relevant $\theta$-stable parabolic subgroups.

Lemma 4.21. Let $\mathbf{P}$ be any $\theta$-stable parabolic subgroup of $\mathbf{G}$, then $P=$ $\mathbf{P}(F)$ is $H$-conjugate to a $\theta$-stable $A_{T}$-semi-standard parabolic subgroup.

Proof. First, note that by Corollary 4.17 and [13, Lemma 3.5], the torus $\left(\mathbf{A}_{T} \cap \mathbf{H}\right)^{\circ}$ is a maximal $F$-split torus of $H$. Let $\mathbf{P}=\mathbf{M N}$ be a $\theta$-stable parabolic subgroup with the indicated Levi factorization, where $\mathbf{M}$ and $\mathbf{N}$ are both $\theta$-stable. Let $\mathbf{P}$. be a minimal $\theta$-stable parabolic subgroup of $\mathbf{G}$ contained in $\mathbf{P}$. Let $\mathbf{A}$. be a $\theta$-stable maximal $F$-split torus contained in P. [13, Lemma 2.5]. By [13, Corollary 5.8], there exists $g=n h \in$ $\left(N_{\mathbf{G}}\left(\mathbf{A}_{\bullet}\right) \cap N_{\mathbf{G}}\left(\left(\mathbf{A}_{\bullet} \cap \mathbf{H}\right)^{\circ}\right)\right)(F) \mathbf{H}(F)$ such that $g^{-1} \mathbf{P} \bullet g=\mathbf{Q}_{0}$. Note that $n$ normalizes $\left(\mathbf{A}_{\bullet} \cap \mathbf{H}\right)^{\circ}$ and all of $\mathbf{A}_{\bullet}$, while $h$ is $\theta$-fixed. Observe that

$$
g^{-1} A_{\bullet} g=h^{-1} n^{-1} A_{\bullet} n h=h^{-1} A_{\bullet} h \subset Q_{0} ;
$$

in particular, $g^{-1} A_{\bullet} g$ is a $\theta$-stable maximal $F$-split torus. Let $\mathbf{U}_{0}$ be the unipotent radical of $\mathbf{Q}_{0}$. By [13, Lemma 2.4], $g^{-1} A \bullet g$ is $\left(\mathbf{H} \cap \mathbf{U}_{0}\right)(F)$ conjugate to $A_{T}$. It follows that there exists $h^{\prime} \in\left(\mathbf{H} \cap \mathbf{U}_{0}\right)(F)$ such that

$$
A_{T}=h^{-1} g^{-1} A_{\bullet} g h^{\prime}=h^{\prime-1} h^{-1} A_{\bullet} h h^{\prime}=\left(h h^{\prime}\right)^{-1} A_{\bullet}\left(h h^{\prime}\right) ;
$$

moreover, we have that $A_{T}=\left(h h^{\prime}\right)^{-1} A_{\bullet}\left(h h^{\prime}\right)$ is contained in $\left(h h^{\prime}\right)^{-1} \mathbf{P}\left(h h^{\prime}\right)$ and $\mathbf{P}$ is $H$-conjugate to a $\theta$-stable $A_{T^{-}}$-semi-standard parabolic subgroup.

Let $n \geq 2$ be an even integer. Let $l=\operatorname{diag}(x, y) \in L=M_{(n / 2, n / 2)}$. We compute that

$$
\theta(l)=w_{\ell}^{-1}\left({ }^{t} \sigma(l)^{-1}\right) w_{\ell}=\left(\begin{array}{cc}
J_{n / 2}^{-1 t} \sigma(y)^{-1} J_{n / 2} & 0 \\
0 & J_{n / 2}^{-1} \sigma(x)^{-1} J_{n / 2}
\end{array}\right) .
$$

It follows immediately from 4.8 that $l$ is $\theta$-fixed if and only if

$$
y=\theta_{J_{n / 2}}(x)=J_{n / 2}^{-1}\left({ }^{t} \sigma(x)^{-1}\right) J_{n / 2},
$$

and observes that,

$$
L^{\theta}=\left\{\left(\begin{array}{cc}
x & 0 \\
0 & \theta_{J_{n / 2}}(x)
\end{array}\right): x \in \mathbf{G L}_{n / 2}(E)\right\} \cong \mathbf{G L}_{n / 2}(E)
$$


From (4.9), we immediately obtain a characterization of the $\theta$-fixed points of the associate Levi subgroup $M={ }^{\gamma} L$ of the $\Delta_{0}$-standard parabolic subgroup $P={ }^{\gamma} Q$.

Lemma 4.22. The Levi subgroup $M={ }^{\gamma} L$ is the $\theta$-stable Levi subgroup of a standard $\theta$-split parabolic $P=M N={ }^{\gamma} P_{(n / 2, n / 2)}$. The $\theta$-fixed points of $M$ are isomorphic to a product of two copies of the unitary group $\mathbf{U}_{E / F, 1_{n / 2}}=$ $\left\{x \in \mathbf{G L}_{n / 2}(E): x^{-1}={ }^{t} \sigma(x)\right\}$, where $1_{n / 2}$ is the $n / 2 \times n / 2$ identity matrix.

Proof. Let ${ }^{\gamma} m \in M$, where $m \in L$. We have that

$$
\begin{aligned}
\theta\left({ }^{\gamma} m\right) & =w_{\ell}^{-1}{ }^{t} \sigma(\gamma)^{-1}{ }^{t} \sigma(m)^{-1}{ }^{t} \sigma(\gamma) w_{\ell} \\
& =\gamma\left({ }^{t} \sigma(\gamma) w_{\ell} \gamma\right)^{-1}{ }^{t} \sigma(m)^{-1}\left({ }^{t} \sigma(\gamma) w_{\ell} \gamma\right) \gamma^{-1} \\
& =\gamma^{t} \sigma(m)^{-1} \gamma^{-1},
\end{aligned}
$$

where the last equality holds since ${ }^{t} \sigma(\gamma) w_{\ell} \gamma \in A_{L}$ centralizes $m \in L$. It follows that ${ }^{\gamma} m=\theta\left({ }^{\gamma} m\right)$ if and only if $m={ }^{t} \sigma(m)^{-1}$. Writing $m$ as a block-diagonal matrix $m=\operatorname{diag}(x, y)$, we have $m={ }^{t} \sigma(m)^{-1}$ if and only if $x={ }^{t} \sigma(x)^{-1}$ and $y={ }^{t} \sigma(y)^{-1}$. It follows that

$$
M^{\theta}=\left\{\gamma\left(\begin{array}{ll}
x & 0 \\
0 & y
\end{array}\right) \gamma^{-1}: x, y \in \mathbf{G L}_{n / 2}(E), x={ }^{t} \sigma(x)^{-1}, y={ }^{t} \sigma(y)^{-1}\right\},
$$

and $M^{\theta} \cong \mathbf{U}_{E / F, 1_{n / 2}} \times \mathbf{U}_{E / F, 1_{n / 2}}$, as claimed.

In Lemma 5.8, we will determine the $\theta$-fixed points of the Levi subgroup of an arbitrary maximal $\theta$-split parabolic subgroup that has $\theta$-stable Levi factor associate to $L$.

\section{Relative Discrete series FOR $\mathbf{U}_{E / F}(F) \backslash \mathbf{G L}_{2 n}(E)$}

From now on, let $G=\mathbf{G L}_{2 n}(E)$, where $n \geq 2$, and let $H=\mathbf{U}_{E / F, w_{\ell}}(F)$ be the $\theta$-fixed points of $G$. Recall that $H$ is (the $F$-points of) a quasi-split unitary group. Let $Q=P_{(n, n)}$ be the type $(n, n)$ block-upper triangular parabolic subgroup of $G$, with block-diagonal Levi factor $L=M_{(n, n)}$ and unipotent radical $U_{(n, n)}$.

In this section we prove Theorem 5.11, the main result of the paper. We construct representations in the discrete series of $H \backslash G$ via parabolic induction from $L^{\theta}$-distinguished discrete series representations of $L$.

The parabolic subgroup $Q$ is conjugate to the $\Delta_{0}$-standard maximal $\theta$ split parabolic $P_{\Omega}$, where $\Omega=\Delta_{0} \backslash\left\{\gamma\left(\epsilon_{n}-\epsilon_{n+1}\right)\right\}$. In particular, $Q=$ $\gamma^{-1} P_{\Omega} \gamma, L=\gamma^{-1} M_{\Omega} \gamma$ and $U=\gamma^{-1} N_{\Omega} \gamma$. For any $\Theta \subset \Delta_{0}$, we use the representatives $\left[W_{\Theta} \backslash W_{0} / W_{\Omega}\right]$ for the double-coset space $P_{\Theta} \backslash G / P_{\Omega}(c f$. Lemma 2.3). There is an isomorphism $P_{\Theta} \backslash G / P_{\Omega} \cong P_{\Theta} \backslash G / Q$ given by $w \mapsto w \gamma$.

5.1. A few more ingredients. Here, we assemble the remaining representation theoretic results needed to state and prove Theorem 5.11. 
5.1.1. The inducing discrete series representations. The irreducible representations distinguished by arbitrary unitary groups are characterized in the paper [8, continuing the work of Jacquet et al., for instance in [15, 16, 18, Feigon, Lapid and Offen study both local and global distinction, largely using global methods. In particular, they show that an irreducible square integrable representation $\pi$ of $G$ is $H^{x}$-distinguished if and only if $\pi$ is Galois invariant. Although Feigon, Lapid and Offen prove much stronger results, we'll recall only what we need for our application. The following appears as [8, Corollary 13.5]. Recall from (4.2) that $X$ is the variety of Hermitian matrices in $\mathbf{G L}_{n}(E)$.

Theorem 5.1 (Feigon-Lapid-Offen). Let $\pi$ be an irreducible admissible essentially square integrable representation of $\mathbf{G L}_{n}(E)$. For any $x \in X$, the following conditions are equivalent:

(1) the representation $\pi$ is Galois invariant, that is $\pi \cong{ }^{\sigma} \pi$.

(2) the representation $\pi$ is $H^{x}$-distinguished.

In addition, $\operatorname{dim} \operatorname{Hom}_{H^{x}}(\pi, 1) \leq 1$.

The multiplicity-one statement appears as [8, Proposition 13.3]. It is known that local multiplicity-one for unitary groups does not hold in general, see [8, Corollary 13.16] for instance, which gives a lower bound for the dimension of $\operatorname{Hom}_{H^{x}}(\pi, 1)$ for Galois invariant generic representations $\pi$. On the other hand, Feigon, Lapid and Offen are able to extend Theorem 5.1 to all ladder representations ( $c f$. [8, Theorem 13.11]).

\subsubsection{Distinction of inducing representations.}

Proposition 5.2. An irreducible admissible representation $\pi_{1} \otimes \pi_{2}$ of $L$ is $L^{\theta}$-distinguished if and only if $\pi_{2}$ is equivalent to the Galois-twist of $\pi_{1}$, that is, if and only if $\pi_{2} \cong{ }^{\sigma} \pi_{1}$.

We actually prove a slightly more general result from which Proposition 5.2 is a trivial corollary, by taking into account the description of $L^{\theta}$ given in 4.9 .

Lemma 5.3. Let $m \geq 1$ be an integer. Let $G^{\prime}=\mathbf{G L}_{m}(E) \times \mathbf{G L}_{m}(E)$, $x \in \mathbf{G L}_{m}(E)$ a Hermitian matrix, and define

$$
H^{\prime}=\left\{\left(\begin{array}{cc}
A & 0 \\
0 & \theta_{x}(A)
\end{array}\right): A \in \mathbf{G L}_{m}(E)\right\} .
$$

An irreducible admissible representation $\pi_{1} \otimes \pi_{2}$ of $G^{\prime}$ is $H^{\prime}$-distinguished if and only if $\pi_{2}$ is equivalent to the Galois-twist of $\pi_{1}$, i.e., $\pi_{2} \cong{ }^{\sigma} \pi_{1}$.

Proof. First, note that a representation $\pi_{1} \otimes \pi_{2}$ is $H^{\prime}$-distinguished if and only if $\pi_{2}$ is equivalent to ${ }^{\theta} \widetilde{\pi}_{1}$, the $\theta_{x}$-twist of the contragredient of $\pi_{1}$. It suffices to show that for any Hermitian matrix $x$ in $\mathbf{G L}_{m}(E)$, and any irreducible admissible representation $\pi$ of $\mathbf{G L}_{m}(E)$, the Galois-twisted representation ${ }^{\sigma} \pi$ is equivalent to ${ }^{\theta_{x}} \widetilde{\pi}$. By a result of Gel'fand and Kazhdan 
[9, Theorem 2], we have that $\widetilde{\pi}$ is equivalent to $\widehat{\pi}$, where the representation $\widehat{\pi}$, is defined by $\widehat{\pi}(g)=\pi\left({ }^{t} g^{-1}\right)$ acting on the space $V$ of $\pi$. Since $\pi$ is admissible, we have $\widetilde{\pi} \cong \pi$; thus, we see that $\widehat{(\widetilde{\pi})} \cong \pi$. On the other hand, the representation $\theta_{x} \widetilde{\pi}$ on $\widetilde{V}$ is given by $\theta_{x} \widetilde{\pi}(g)=\tilde{\pi}\left(\theta_{x}(g)\right)$. Using that $x$ is Hermitian, it is readily verified that

$$
\theta_{x} \widetilde{\pi}(g)={ }^{\sigma} \widehat{(\widetilde{\pi})}\left(x g x^{-1}\right)={ }^{-1}(\widehat{\sigma}(\widehat{\widetilde{\pi}}))(g) .
$$

We observe that ${ }^{\theta_{x}} \widetilde{\pi}$ is equivalent to $\sigma \widehat{(\widetilde{\pi})}$ since Int $x^{-1}$ is an inner automorphism of $\mathbf{G L}_{m}(E)$. It is also clear that taking Galois twists commutes with the map sending $\pi$ to $\widehat{\pi}$ (and twisting by $\operatorname{Int} x^{-1}$, since $x$ is Hermitian). Finally, we have shown that

$$
\theta_{x} \widetilde{\pi} \cong x^{-1}(\widehat{\sigma}(\widehat{\tilde{\pi}})) \cong \sigma \widehat{(\widetilde{\pi})} \cong{ }^{\sigma} \pi,
$$

as claimed.

5.1.3. H-distinction of an induced representation. Let $\tau^{\prime}$ be an irreducible admissible representation of $\mathbf{G L}_{n}(E)$ and define $\tau=\tau^{\prime} \otimes{ }^{\sigma} \tau^{\prime}$. By Proposition 5.2, the irreducible admissible representation $\tau$ of $L$ is $L^{\theta}$-distinguished. Let $\lambda$ be a nonzero element of $\operatorname{Hom}_{L^{\theta}}(\tau, 1)$. The invariant form $\lambda$ is defined using the pairing of $\tau^{\prime}$ with its contragredient. By [23, Proposition 4.3.2], we have that $\delta_{Q}^{1 / 2}$ restricted to $L^{\theta}$ is equal to $\delta_{Q \cap H}=\delta_{Q^{\theta}}$. By Corollary 2.8. we have the following result.

Proposition 5.4. Let $\tau^{\prime}$ be an irreducible admissible representation of $\mathbf{G L}_{n}(E)$. If $\tau=\tau^{\prime} \otimes{ }^{\sigma} \tau^{\prime}$, then the induced representation $\pi=\iota_{Q}^{G} \tau$ is H-distinguished.

5.2. Computing exponents and distinction of Jacquet modules. Let $P=M N$ be a proper $\theta$-split parabolic subgroup of $G$, with $\theta$-stable Levi factor $M$ and unipotent radical $N$. By the Geometric Lemma (Lemma 2.1) and Lemma 3.1, the exponents of $\pi=\iota_{Q}^{G} \tau$ along $P$ are given by

$$
\operatorname{Exp}_{A_{M}}\left(\pi_{N}\right)=\bigcup_{y \in M \backslash S(M, L) / L} \operatorname{Exp}_{A_{M}}\left(\mathcal{F}_{N}^{y}(\tau)\right),
$$

and the exponents $\mathscr{E} x p_{A_{M}}\left(\mathscr{F}_{N}^{y}(\tau)\right)$ are the central characters of the irreducible subquotients of the representations $\mathscr{F}_{N}^{y}(\tau)$ ( $c f$. Remark 2.2). Note that restriction of characters from $A_{M}$ to the $(\theta, F)$-split component $S_{M}$ provides a surjection from $\mathscr{E} x p_{A_{M}}\left(\pi_{N}\right)$ to $\mathscr{E} x p_{S_{M}}\left(\pi_{N}\right)$, for instance see [29, Lemma 4.15].

Let $y \in M \backslash S(M, L) / L$. There are two situations that we need to consider:

Case (1): when $P \cap{ }^{y} L={ }^{y} L$, and

Case (2): when $P \cap{ }^{y} L \subsetneq{ }^{y} L$ is a proper parabolic subgroup of ${ }^{y} L$.

By Lemma 4.3, there exists a $\theta$-split subset $\Theta \subset \Delta_{0}$ and an element $g \in\left(\mathbf{T}_{\mathbf{0}} \mathbf{H}\right)(F)$ such that $P=g P_{\Theta} g^{-1}$. We choose a representative of $y$ with the form $y=g w \gamma$, where $w \in\left[W_{\Theta} \backslash W_{0} / W_{\Omega}\right]$. Recall that $Q=\gamma^{-1} P_{\Omega} \gamma$, $U=\gamma^{-1} N_{\Omega} \gamma$, and $L=\gamma^{-1} M_{\Omega} \gamma$, where $\Omega=\Delta_{0} \backslash\left\{\gamma\left(\epsilon_{n}-\epsilon_{n+1}\right)\right\}$. 
5.2.1. Case (1). Suppose that $P \cap{ }^{y} L={ }^{y} L$. Then $M \cap{ }^{y} L={ }^{y} L \cong M_{(n, n)}$. Moreover, the Levi subgroup $M$ must be a maximal proper Levi subgroup of $G$ that is associate to $L$. It follows that, in this case, $\Theta=\Omega$.

There are exactly two elements $w \in\left[W_{\Omega} \backslash W_{0} / W_{\Omega}\right]$ such that $y=g w \gamma$ satisfies $M \cap{ }^{y} L={ }^{y} L$ : the identity and ${ }^{\gamma} w_{L}$, where

$$
w_{L}=\left(\begin{array}{cc}
0 & 1_{n} \\
1_{n} & 0
\end{array}\right) \in N_{G}(L),
$$

and $1_{n}$ is the $n \times n$ identity matrix. It follows that, in Case (1), there are two elements $y$ that we need to consider: $y=g e \gamma=g \gamma$ and $y=g^{\gamma} w_{L} \gamma=g \gamma w_{L}$.

Note. For a representation $\tau^{\prime}$ of $\mathbf{G L}_{n}(E)$, we have ${ }^{w_{L}}\left(\tau^{\prime} \otimes{ }^{\sigma} \tau^{\prime}\right) \cong{ }^{\sigma} \tau^{\prime} \otimes \tau^{\prime}$

We obtain the following.

Lemma 5.5. Let $\tau=\tau^{\prime} \otimes{ }^{\sigma} \tau^{\prime}$ be an irreducible admissible representation of L. Let $P={ }^{g} P_{\Omega}, g \in\left(\mathbf{T}_{\mathbf{0}} \mathbf{H}\right)(F)$, be a maximal $\theta$-split parabolic subgroup with Levi associate to L. Let $\pi=\iota_{Q}^{G} \tau$.

(1) If $y=g \gamma$, then $\mathcal{F}_{N}^{y}(\tau)={ }^{g \gamma} \tau={ }^{g \gamma}\left(\tau^{\prime} \otimes{ }^{\sigma} \tau^{\prime}\right)$.

(2) If $y=g \gamma w_{L}$, then $\mathscr{F}_{N}^{y}(\tau)={ }^{g \gamma w_{L}} \tau={ }^{g \gamma}\left({ }^{\sigma} \tau^{\prime} \otimes \tau^{\prime}\right)$

Proof. Indeed, for $y=g \gamma x$, where $x$ normalizes $L$, we have

$$
M \cap{ }^{y} Q=M \cap g \gamma Q \gamma^{-1} g^{-1}=M \cap P=M
$$

and

$$
P \cap{ }^{y} L=P \cap g \gamma L \gamma^{-1} g^{-1}=P \cap M=M={ }^{y} L,
$$

so we have that

$$
\mathcal{F}_{N}^{y}(\tau)=\iota_{M}^{M}\left(\left({ }^{y} \tau\right)_{\{e\}}\right)={ }^{y} \tau \text {. }
$$

If $\tau$ is an irreducible unitary (e.g., a discrete series) representation of $L$, then the two subquotients $\mathscr{F}_{N}^{g \gamma}(\tau)={ }^{g \gamma}\left(\tau^{\prime} \otimes{ }^{\sigma} \tau^{\prime}\right)$ and $\mathscr{F}_{N}^{g \gamma w_{L}}(\tau)={ }^{g \gamma}\left({ }^{\sigma} \tau^{\prime} \otimes \tau^{\prime}\right)$ of $\pi_{N}$ are irreducible and unitary.

5.2.2. Case (2). Suppose that $P \cap^{y} L \subsetneq^{y} L$ is a proper parabolic subgroup of ${ }^{y} L$. In particular, the Levi subgroup $M \cap{ }^{y} L$ of $P \cap{ }^{y} L$ is properly contained in ${ }^{y} L$. The following is the direct analogue of [29, Proposition 8.5]. The idea of the proof is to realize the exponents of $\pi$ along $P$ as restrictions of the exponents of $\tau$ along $P \cap y$ ( $c f$. [29, Lemma 4.16]) and to apply Casselman's Criterion to the discrete series $\tau$.

Proposition 5.6. Let $P$ be a maximal $\theta$-split parabolic subgroup of $G$ and $y \in P \backslash G / Q$ such that $P \cap{ }^{y} L$ is a proper parabolic subgroup of ${ }^{y} L$. Let $\tau$ be a discrete series representation of $L$. The exponents of $\pi=\iota_{Q}^{G} \tau$ along $P$ contributed by the subquotient $\mathscr{F}_{N}^{y}(\tau)=\iota_{M \cap^{y} Q}^{M}\left({ }^{y} \tau\right)_{N \cap^{y} L}$ of $\pi_{N}$ satisfy the condition in 3.2 .

\footnotetext{
${ }^{4}$ The Weyl group element $w_{L}$ has the property that ${ }^{w_{L}} Q=Q^{\mathrm{op}}$.
} 
Proof. If $\tau$ is a discrete series representation of $L$, then $\tau$ satisfies Casselman's Criterion ( $c f$. [6, Theorem 6.5.1]). In light of Lemma 6.2, the argument that the exponents of $\pi=\iota_{Q}^{G} \tau$ along $P$ satisfy the Relative Casselman's Criterion (Theorem 3.4) follows exactly as in the proof of [29, Proposition 8.5].

5.2.3. Distinction of $\mathcal{F}_{N}^{y}(\tau)$ and $\pi_{N}$. A consequence of Proposition 5.6 is that we need only consider distinction of (subquotients of) the Jacquet module of $\pi=\iota_{Q}^{G} \tau$ in Case (1) (cf. Theorem 3.4 and Proposition 3.5). We now determine the $\theta$-fixed points of $M$ and study $M^{\theta}$-distinction of the irreducible subquotients of $\pi_{N}$ in the case that $P \cap{ }^{y} L={ }^{y} L$.

Lemma 5.7. The intersection $N_{G}(L) \cap Q^{\mathrm{op}}$ of the normalizer of $L$ in $G$ with the opposite parabolic $Q^{\mathrm{op}}$ of $Q$ is equal to $L$.

Proof. Suppose that $q=\left(\begin{array}{cc}A & 0 \\ B & C\end{array}\right) \in N_{G}(L) \cap Q^{o p}$, and let $l=\operatorname{diag}\left(l_{1}, l_{2}\right)$ be an arbitrary element of $L$. We have that

$$
q l q^{-1}=\left(\begin{array}{cc}
A l_{1} A^{-1} & 0 \\
B l_{1} A^{-1}-C l_{2} C^{-1} B A^{-1} & C l_{2} C^{-1}
\end{array}\right) \in L,
$$

and we see that

$$
B l_{1} A^{-1}-C l_{2} C^{-1} B A^{-1}=0,
$$

for all $l_{1}, l_{2} \in \mathbf{G L}_{n}(E)$. This occurs if and only if $B=C l_{2} C^{-1} B l_{1}^{-1}$ for all $l_{1}, l_{2} \in \mathbf{G L}_{n}(E)$. If we take $l_{2}=1_{n}$ to be the identity, then $B l_{1}=B$ for any $l_{1} \in \mathbf{G L}_{n}(E)$, which occurs if and only if $B\left(l_{1}-1_{n}\right)=0$ for any $l_{1} \in \mathbf{G L}_{n}(E)$. In particular, since there exists $l_{1} \in \mathbf{G L}_{n}(E)$ such that $l_{1}-1_{n}$ is invertible, we must have that $B=0$. The lemma follows.

Lemma 5.8. Let $M=g \gamma M_{(n, n)} \gamma^{-1} g^{-1}$, where $g \in\left(\mathbf{H T}_{0}\right)(F)$.

(1) The subgroup $M^{\theta}$ of $\theta$-fixed points in $M$ is the $g \gamma$-conjugate of $L^{g \gamma \cdot \theta}$.

(2) We have that $L^{g \gamma \cdot \theta}=L^{\theta_{x_{L}}}$ is equal to the product $\mathbf{U}_{E / F, x_{1}} \times \mathbf{U}_{E / F, x_{2}}$ of unitary groups.

(3) Explicitly, $M^{\theta}=g \gamma\left(\mathbf{U}_{E / F, x_{1}} \times \mathbf{U}_{E / F, x_{2}}\right) \gamma^{-1} g^{-1}$ is isomorphic to a product of unitary groups.

(4) Let $\tau$ be an irreducible admissible representation of $L$. Then ${ }^{g \gamma} \tau$ is $M^{\theta}$-distinguished if and only if $\tau$ is $\mathbf{U}_{E / F, x_{1}} \times \mathbf{U}_{E / F, x_{2}}$-distinguished.

Proof. By Lemma 5.7, we have that $x_{L}=\gamma^{-1} g^{-1} \theta(g) \gamma=\operatorname{diag}\left(x_{1}, x_{2}\right) \in L$; moreover, $x_{L}$ is Hermitian. Indeed, since ${ }^{t} \gamma=\gamma=\sigma(\gamma)$ and $w_{\ell}={ }^{t} w_{\ell}=$ 
$\sigma\left(w_{\ell}\right)$, we have

$$
\begin{aligned}
{ }^{t} \sigma\left(x_{L}\right) & ={ }^{t} \sigma\left(\gamma^{-1} g^{-1} \theta(g) \gamma\right) \\
& ={ }^{t} \sigma\left(\gamma^{-1} g^{-1} w_{\ell}^{-1}\left({ }^{t} \sigma(g)^{-1}\right) w_{\ell} \gamma\right) \\
& =\sigma\left({ }^{t} \gamma\right) \sigma\left({ }^{t} w_{\ell}\right) g^{-1} \sigma\left({ }^{t} w_{\ell}\right)^{-1} \sigma\left({ }^{t} g\right)^{-1} \sigma\left({ }^{t} \gamma\right)^{-1} \\
& =\gamma w_{\ell} g^{-1} w_{\ell}^{-1 t} \sigma(g)^{-1} \gamma^{-1} \\
& =\gamma w_{\ell} g^{-1} w_{\ell}^{-1 t} \sigma(g)^{-1} w_{\ell} w_{\ell}^{-1} \gamma^{-1} \\
& =\gamma w_{\ell} g^{-1} \theta(g) w_{\ell}^{-1} \gamma^{-1} \\
& =z \gamma^{-1} g^{-1} \theta(g) \gamma z^{-1} \\
& =z x_{L} z^{-1} \\
& =x_{L}
\end{aligned}
$$

where $z={ }^{t} \sigma(\gamma) w_{\ell} \gamma=\gamma w_{\ell} \gamma \in A_{L}$. In fact, we have that $x_{1}$ and $x_{2}$ are Hermitian elements of $\mathbf{G L}_{n}(E)$.

Upon restriction to $L, g \gamma \cdot \theta=x_{L} \cdot \theta_{e}=\operatorname{Int} x_{L}^{-1} \circ{ }^{t} \sigma()^{-1}$. Note also that $x_{L} \cdot \theta_{e}=\theta_{e \cdot x_{L}}=\theta_{x_{L}}$. In particular, $l \in L$ is $g \gamma \cdot \theta$-fixed if and only if $l$ is $\theta_{x_{L}}$ fixed. Explicitly, $l \in L$ is $\theta_{x_{L}}$-fixed if and only if $l=x^{-1 t} \sigma(l)^{-1} x_{L}$. Since $x_{L}$ is Hermitian, we have that $L^{g \gamma \cdot \theta}=L^{\theta_{x_{L}}}$ is equal to the product $\mathbf{U}_{E / F, x_{1}} \times$ $\mathbf{U}_{E / F, x_{2}}$ of unitary groups. Now, observe that $M^{\theta}=g \gamma L^{g \gamma \cdot \theta}(g \gamma)^{-1}$. Indeed, if $m=g \gamma l \gamma^{-1} g^{-1} \in M$, where $l \in L$, then $m$ is $\theta$-fixed if and only if $l=(g \gamma \cdot \theta)(l)$ is $(g \gamma \cdot \theta)$-fixed.

It is interesting to note that, even though we're interested in distinction by the quasi-split unitary group $\mathbf{H}=\mathbf{U}_{E / F, w_{\ell}}$, we will need to consider distinction by (possibly non-quasi-split) unitary groups for Jacquet modules.

Define a representation $\rho$ of a Levi subgroup $M$ of $G$ to be regular if for every non-trivial element $w \in N_{G}(M) / M$ we have that the twist ${ }^{w} \rho=$ $\rho\left(w^{-1}(\cdot) w\right)$ is not equivalent to $\rho$. A representation $\pi_{1} \otimes \pi_{2}$ of $L$ is regular if and only if $\pi_{1} \neq \pi_{2}$. The next lemma is [29, Lemma 8.2].

Lemma 5.9. Assume that $\tau$ is a regular unitary irreducible admissible representation of $L$. Let $P=M N$ be a $\theta$-split parabolic subgroup with Levi associate to L. If $y \in M \backslash S(M, L) / L$ is such that $P \cap{ }^{y} L={ }^{y} L$, then $\mathcal{F}_{N}^{y}(\tau)$ is irreducible and the central character $\chi_{N, y}$ of $\mathscr{F}_{N}^{y}(\tau)$ is unitary.

From Lemma 5.5. it follows that $M^{\theta}$-distinction of ${ }^{g \gamma} \tau$ (respectively, $\left.{ }^{g \gamma w_{L}} \tau\right)$ is equivalent to $\mathbf{U}_{E / F, x_{1}}$-distinction of $\tau^{\prime}$ and $\mathbf{U}_{E / F, x_{2}}$-distinction of ${ }^{\sigma} \tau^{\prime}$ (respectively, $\mathbf{U}_{E / F, x_{1}}$-distinction of ${ }^{\sigma} \tau^{\prime}$ and $\mathbf{U}_{E / F, x_{2}}$-distinction of $\tau^{\prime}$ ). If $\tau=\tau^{\prime} \otimes{ }^{\sigma} \tau^{\prime}$ is a regular discrete series representation, then $\tau^{\prime} ¥{ }^{\sigma} \tau^{\prime}$. It follows from Theorem 5.1 that neither $\tau^{\prime}$ nor ${ }^{\sigma} \tau^{\prime}$ can be distinguished by any unitary group. If $P=M N$ is any $\theta$-split parabolic such that $M$ is associate to $L$, then by Lemma 5.8, neither of the irreducible subquotients of $\pi_{N}$, described in Lemma 5.5, can be $M^{\theta}$-distinguished. We records this as the following. 
Corollary 5.10. Let $\pi=\iota_{Q}^{G} \tau$, where $\tau=\tau^{\prime} \otimes{ }^{\sigma} \tau^{\prime}$ is a discrete series representation such that $\tau^{\prime} \not{ }^{\sigma} \tau^{\prime}$. Let $P={ }^{g} P_{\Omega}$, where $g \in\left(\mathbf{H T}_{0}\right)(F)$, be any maximal $\theta$-split parabolic subgroup with $\theta$-stable Levi $M=P \cap \theta(P)$ associate to $L$. Neither of the two irreducible unitary subquotients of $\pi_{N}$, twists of $\tau^{\prime} \otimes{ }^{\sigma} \tau^{\prime}$ and ${ }^{\sigma} \tau^{\prime} \otimes \tau^{\prime}$ (cf. Lemma 5.5), can be $M^{\theta}$-distinguished.

5.3. Constructing relative discrete series. The following theorem is our main result. The argument is the same as the proof of [30, Theorem 6.3].

Theorem 5.11. Let $Q=P_{(n, n)}$ be the upper-triangular parabolic subgroup of $G$ with standard Levi factor $L=M_{(n, n)}$ and unipotent radical $U=N_{(n, n)}$. Let $\pi=\iota_{Q}^{G} \tau$, where $\tau=\tau^{\prime} \otimes{ }^{\sigma} \tau^{\prime}$, and $\tau^{\prime}$ is a discrete series representation of $\mathbf{G L}_{n}(E)$ such that $\tau^{\prime}$ is not Galois invariant, i.e., $\tau^{\prime} ¥{ }^{\sigma} \tau^{\prime}$. The representation $\pi$ is a relative discrete series representation for $H \backslash G$ that does not occur in the discrete series of $G$.

Proof. Since $\tau$ is unitary and regular, by result of Bruhat [5] (cf. [6, Theorem 6.6.1]), $\pi$ is irreducible. In addition, $\pi$ is $H$-distinguished by Proposition 5.4. The representation $\pi$ does not occur in the discrete series of $G$ by Zelevinsky's classification [31. Let $\lambda$ denote a fixed $H$-invariant linear form on $\pi$. It suffices to show that $\pi$ satisfies the Relative Casselman's Criterion Theorem 3.4. Let $P=M N$ be a proper $\theta$-split parabolic subgroup of $G$. The exponents of $\pi$ along $P$ are the central characters of the irreducible subquotients of the representations $\mathscr{F}_{N}^{y}(\tau)$ given by the Geometric Lemma 2.1 (see Section 5.2]. By [21, Lemma 4.6] and Proposition 5.6, the condition $(3.2)$ is satisfied when $P \cap{ }^{y} L$ is a proper parabolic subgroup of ${ }^{y} L$. As in Lemma 5.9, the only unitary exponents of $\pi$ along $P$ occur when $P \cap{ }^{y} L={ }^{y} L$. By Corollary 5.10, the only irreducible unitary subquotients of $\pi_{N}$ cannot be $M^{\theta}$-distinguished when $P \cap{ }^{y} L={ }^{y} L$. In the latter case, by Proposition 3.5, the unitary exponents of $\pi$ along $P$ do not contribute to $\varepsilon_{x} p_{S_{M}}\left(\pi_{N}, \lambda_{N}\right)$. Therefore, 3.2 is satisfied for every proper $\theta$-split parabolic subgroup of $G$. Finally, by Theorem 3.4 the representation $\pi$ appears in the discrete spectrum of $H \backslash G$. In particular, $\pi$ is $(H, \lambda)$-relatively square integrable for all nonzero $\lambda \in \operatorname{Hom}_{H}(\pi, 1)$.

In addition, we note the following existence results. First, we recall the structure of the representations in the discrete spectrum of $\mathbf{G L}_{n}(E)$. Let $\rho$ be an irreducible unitary supercuspidal representation of $\mathbf{G L}_{r}(E), r \geq 1$. For an integer $k \geq 2$, write $\operatorname{St}(k, \rho)$ for the unique irreducible (unitary) quotient of the parabolically induced representation

$$
\iota_{P_{(r, \ldots, r)}}^{\mathbf{G L}_{k r}(E)}\left(\nu^{\frac{1-k}{2}} \rho \otimes \nu^{\frac{3-k}{2}} \rho \otimes \ldots \otimes \nu^{\frac{k-1}{2}} \rho\right)
$$

of $\mathbf{G L}_{k r}(E)$ ( $c f$. [31, Proposition 2.10, $\left.\S 9.1\right]$ ), where $\nu(g)=|\operatorname{det}(g)|_{E}$, for any $g \in \mathbf{G L}_{r}(E)$. The representations $\operatorname{St}(k, \rho)$ are the generalized Steinberg representations and they are precisely the nonsupercuspidal discrete series representations of $\mathbf{G L}_{k r}(E)$ [31, Theorem 9.3]. The usual Steinberg representation $\mathrm{St}_{n}$ of $\mathbf{G L}_{n}(E)$ is obtained as $\operatorname{St}(n, 1)$. 
Proposition 5.12. Let $n \geq 2$ be an integer. There exist infinitely many equivalence classes of non-supercuspidal discrete series representations $\tau$ of $\mathbf{G L}_{n}(E)$ that are not Galois invariant.

Before giving a proof of Proposition 5.12, we note the following results.

Corollary 5.13. Let $n \geq 4$ be an integer. There exist infinitely many equivalence classes of $R D S$ representations of the form constructed in Theorem 5.11 and such that the discrete series $\tau$ is not supercuspidal.

Proof. Apply Proposition 5.12 and [31, Theorem 9.7(b)].

Proposition 5.14. Let $\rho$ be an irreducible supercuspidal representation of $\mathbf{G L}_{r}(E), r \geq 1$. For $k \geq 2$, the generalized Steinberg representation $\operatorname{St}(k, \rho)$ of $\mathbf{G L}_{k r}(E)$ is Galois invariant if and only if $\rho$ is Galois invariant.

Proof. First, observe that the twisted representation ${ }^{\sigma} \operatorname{St}(k, \rho)$ is equivalent to the generalized Steinberg representation $\operatorname{St}\left(k,{ }^{\sigma} \rho\right)$. It follows that $\operatorname{St}(k, \rho)$ is Galois invariant if and only if $\operatorname{St}(k, \rho) \cong{ }^{\sigma} \operatorname{St}(k, \rho) \cong \operatorname{St}\left(k,{ }^{\sigma} \rho\right)$. The result now follows from [31, Theorem 9.7(b)], which gives us that $\operatorname{St}(k, \rho) \cong$ $\operatorname{St}\left(k,{ }^{\sigma} \rho\right)$ if and only if $\rho \cong{ }^{\sigma} \rho$.

Proposition 5.15. For $n \geq 2$, there exist infinitely many unitary twists of the Steinberg representation $\mathrm{St}_{n}$ of $\mathbf{G L}_{n}(E)$ that are not Galois invariant.

Proof. Let $\chi: E^{\times} \rightarrow \mathbb{C}^{\times}$be a (unitary) character of $E^{\times}$. By [31, Theorem $9.7(\mathrm{~b})], \chi \mathrm{St}_{n} \cong \sigma\left(\chi \mathrm{St}_{n}\right)$ if and only if $\chi={ }^{\sigma} \chi$. We have that ${ }^{\sigma} \chi=\chi$ if and only if $\chi$ is trivial on the kernel of the norm map $N_{E / F}: E^{\times} \rightarrow F^{\times}$. Note that ker $N_{E / F}$ is a non-trivial closed subgroup of $E^{\times}$. We can extend any non-trivial unitary character of ker $N_{E / F}$ to $E^{\times}$to obtain a unitary character $\chi$ of $E^{\times}$such that ${ }^{\sigma} \chi \neq \chi$. Given a unitary character of ker $N_{E / F}$ there are infinitely many distinct extensions to $E^{\times}$.

The following is the main ingredient needed to prove Proposition 5.12.

Theorem 5.16 (Hakim-Murnaghan). For $n \geq 1$, there exist infinitely many distinct equivalence classes of Galois invariant (respectively, non-Galois invariant) irreducible supercuspidal representations of $\mathbf{G L}_{n}(E)$.

Proof. If $n=1$, argue as in the proof of Proposition 5.15. For $n \geq 2$, use [24, Proposition 10.1] to obtain the existence of infinitely many pairwise inequivalent Galois invariant irreducible supercuspidal representations of $\mathbf{G L}_{n}(E)$. To complete the proof, apply [11, Theorem 1.1].

Proof of Proposition 5.12. If $n$ is prime, then by Proposition 5.15 there exist infinitely many twists of the Steinberg representation $\mathrm{St}_{n}$ of $\mathbf{G L}_{n}(E)$ that are not Galois invariant. If $n$ is composite, then Proposition 5.15 still applies; however, by Proposition 5.14 and Theorem 5.16 there are infinitely many classes of non-Galois invariant generalized Steinberg representations of $\mathbf{G L}_{n}(E)$. 
Remark 5.17. A representation $(\pi, V)$ is $H$-relatively supercuspidal if and only if the $\lambda$-relative matrix coefficients of $\pi$ are compactly supported modulo $Z_{G} H$, for every nonzero $\lambda \in \operatorname{Hom}_{H}(\pi, 1)$. If further assume that $\tau^{\prime}$ is supercuspidal in Theorem 5.11, then $\pi=\iota_{Q}^{G} \tau$ is a non-supercuspidal $H$-relatively supercuspidal representation of $G$ ( $c f$. [29, Corollary 6.7]). A proof of this result can be obtained by a slight modification to proof of Theorem 5.11. Indeed, when $P \cap{ }^{y} L$ is proper in ${ }^{y} L$, the subquotients $\mathscr{F}_{N}^{y}(\tau)$ of the Jacquet module vanish since $\tau$ is supercuspidal. Otherwise, $\mathscr{F}_{N}^{y}(\tau)$ cannot be $M^{\theta}$-distinguished. By Proposition 3.5, $\lambda_{N}=0$, for every proper $\theta$-split parabolic subgroup $P$ of $G$ and any $\lambda \in \operatorname{Hom}_{H}(\pi, 1)$. Finally, by a result of Kato and Takano [20, Theorem 6.2], $\pi$ is relatively supercuspidal. Moreover, since $\pi$ is parabolically induced, $\pi$ is not supercuspidal. This modification of Theorem 5.11 can be obtained by more direct methods; see, for instance, the work of Murnaghan [25].

5.4. Exhaustion of the discrete spectrum. In his 2017 Cours Peccot, Raphaël Beuzart-Plessis announced Plancherel formulas for the two $p$-adic symmetric spaces $\mathbf{G L}_{n}(F) \backslash \mathbf{G L}_{n}(E)$ and $\mathbf{U}_{n, E / F}(F) \backslash \mathbf{G L}_{n}(E)$, where $\mathbf{U}_{n, E / F}(F)$ is a quasi-split unitary group [4]. The two Plancherel formulas are realized in terms of the appropriate base change maps. Both results are obtained by a comparison of local relative trace formulas. Presently, we are concerned with the second case and only when $n$ is even.

As above, let $G=\mathbf{G L}_{2 n}(E)$ and $H=\mathbf{U}_{E / F, w_{\ell}}(F)$, where $n \geq 2$. Building on the work of Jacquet [16] and Feigon-Lapid-Offen [8], Beuzart-Plessis has shown that the Plancherel formula for $H \backslash G$ is the push-forward of the Whittaker-Plancherel formula for $\mathbf{G L}_{2 n}(F)$ via quadratic base change. As a consequence, the discrete spectrum of $H \backslash G$ consists of the quadratic base changes of the discrete series of $\mathbf{G L}_{2 n}(F)$.

Let $\operatorname{Irr}\left(\mathbf{G L}_{n}(E)\right)$ denote the set of equivalence classes of irreducible admissible representations of $\mathbf{G} \mathbf{L}_{n}(E)$ (likewise for $\mathbf{G} \mathbf{L}_{n}(F)$ ), and let $\operatorname{Irr}^{\sigma}\left(\mathbf{G L}_{n}(E)\right.$ ) denote the subset $\left\{\pi \in \operatorname{Irr}\left(\mathbf{G L}_{n}(E)\right): \pi \cong{ }^{\sigma} \pi\right\}$ of Galois invariant representations. Quadratic base change bc $: \operatorname{Irr}\left(\mathbf{G L}_{n}(F)\right) \rightarrow \operatorname{Irr}^{\sigma}\left(\mathbf{G L}_{n}(E)\right)$ maps (classes of) irreducible representations of $\mathbf{G L}_{n}(F)$ to (classes of) irreducible Galois invariant representations of $\mathbf{G L}_{n}(E)$. Moreover, the map bc $: \operatorname{Irr}\left(\mathbf{G L}_{n}(F)\right) \rightarrow \operatorname{Irr}^{\sigma}\left(\mathbf{G L}_{n}(E)\right)$ is surjective. Let $\eta_{E / F}: F^{\times} \rightarrow \mathbb{C}^{\times}$be the quadratic character associated to the extension $E / F$ by local class field theory. For any $\pi^{\prime} \in \operatorname{Irr}\left(\mathbf{G L}_{n}(F)\right)$, we have that $\mathrm{bc}\left(\pi^{\prime}\right)=\mathrm{bc}\left(\pi^{\prime} \otimes \eta_{E / F}\right)$. We refer the reader to [1, Chapter 1, Section 6] for more information about quadratic base change and its properties; in particular, [1, Chapter 1, Theorem 6.2 ] summarizes the basic properties of base change for tempered representations.

In the language of [8], the RDS representations $\pi=\iota_{Q}^{G}\left(\tau^{\prime} \otimes{ }^{\sigma} \tau^{\prime}\right)$, with $\tau^{\prime} ¥{ }^{\sigma} \tau^{\prime}$, constructed in Theorem 5.11 are totally $\sigma$-isotropic, that is, the cuspidal support of $\pi$ is a tensor product of non-Galois invariant supercuspidal representations (see Proposition 5.14). Moreover, this means that $\pi$ is 
the base change of a unique discrete series representation $\pi^{\prime} \cong \pi^{\prime} \otimes \eta_{E / F}$ of $\mathbf{G L}_{2 n}(F)$, and $\pi$ is not distinguished by the non-quasi-split unitary group [8, Theorem 0.2, Lemma 3.4].

The following theorem frames the work of Beuzart-Plessis in terms of Theorem 5.11 and gives a complete description of $L_{\text {disc }}^{2}(H \backslash G)$.

Theorem 5.18. Let $\pi$ be a relative discrete series representation for the quotient $\mathbf{U}_{E / F}(F) \backslash \mathbf{G L}_{2 n}(E)$. Then $\pi$ is either an $\mathbf{U}_{E / F}(F)$-distinguished discrete series representation of $\mathbf{G L}_{2 n}(E)$, or $\pi$ is equivalent to a representation of the form constructed in Theorem 5.11.

Proof. Beuzart-Plessis has shown that the relative discrete series representations for $H \backslash G$ are precisely the images of the discrete series of $\mathbf{G L}_{2 n}(F)$ under quadratic base change [4]. Let $\pi^{\prime} \in \operatorname{Irr}\left(\mathbf{G L}_{2 n}(F)\right)$ be a discrete series representation of $\mathbf{G L}_{2 n}(F)$. By [1, Proposition 6.6], $\pi=\mathrm{bc}\left(\pi^{\prime}\right) \in \operatorname{Irr}^{\sigma}(G)$ is a discrete series representation of $G$ if and only if $\pi^{\prime} \not \pi^{\prime} \otimes \eta_{E / F}$. In this case, $\pi=\operatorname{bc}\left(\pi^{\prime}\right)$ is an $H$-distinguished discrete series representation of $G[8$, Corollary 13.5] and $\pi$ is known to be relatively discrete [21, Proposition 4.10]. Otherwise, it must be the case that $\pi^{\prime} \cong \pi^{\prime} \otimes \eta_{E / F}$. If $\pi^{\prime} \in \operatorname{Irr}\left(\mathbf{G L}_{2 n}(F)\right)$ is a discrete series representation such that $\pi^{\prime} \cong \pi^{\prime} \otimes \eta_{E / F}$, then there exists a (non-unique) discrete series representation $\tau^{\prime} \in \operatorname{Irr}\left(\mathbf{G L}_{n}(E)\right)$ such that $\tau^{\prime} ¥{ }^{\sigma} \tau^{\prime}$ and $\operatorname{bc}\left(\pi^{\prime}\right)=\iota_{P_{(n, n)}}^{G}\left(\tau^{\prime} \otimes{ }^{\sigma} \tau^{\prime}\right)$ is equivalent to a relative discrete series representation constructed in Theorem 5.11 (cf. [8, Section 3.2, Lemma $3.4]) !^{5}$

\section{A technical Lemma}

Finally, we give two technical results required to prove Proposition 5.6, which allows us to reduce the Relative Casselman's Criterion for $\pi=\iota_{Q}^{G} \tau$ to the usual Casselman's Criterion for $\tau$. The set $A_{M \cap^{y} L}^{-y_{L}} \backslash A_{M \cap^{y}{ }{ }^{A} y_{L}}^{1}$ that appears in Lemma 6.2 is the dominant part of $A_{M \cap \cap_{L} L}$ in $M \cap{ }^{y} L$, and is precisely the cone on which we must consider the exponents of $\tau$ in order to apply Casselman's Criterion. Lemma 6.1 is the analogue of [29, Lemma 8.4] and the proof is essentially the same ( $c f$. [30, Lemma 5.2.13]). In the present setting, we must also consider non- $\Delta_{0}$-standard $\theta$-split parabolic subgroups in our analysis of the exponents of $\pi$. In Lemma 6.2, we will explain how to adapt Lemma 6.1 to handle the non-standard case.

In order to discuss Casselman's Criterion for the inducing data of $\pi=\iota_{Q}^{G} \tau$ we use the following notation. If $\Theta_{1} \subset \Theta_{2} \subset \Delta_{0}$, then we define

$$
A_{\Theta_{1}}^{-}=\left\{a \in A_{\Theta_{1}}:|\alpha(a)| \leq 1, \text { for all } \alpha \in \Delta_{0} \backslash \Theta_{1}\right\}
$$

and

$$
A_{\Theta_{1}}^{-\Theta_{2}}=\left\{a \in A_{\Theta_{1}}:|\beta(a)| \leq 1, \text { for all } \beta \in \Theta_{2} \backslash \Theta_{1}\right\} .
$$

\footnotetext{
${ }^{5}$ In this case, the representation $\pi^{\prime}$ is the automorphic induction of the discrete series $\tau^{\prime}$, see [8, Section 3.2].
} 
The set $A_{\Theta_{1}}^{-}$is the dominant part of $A_{\Theta_{1}}$ in $G$, while $A_{\Theta_{1}}^{-\Theta_{2}}$ is the dominant part of $A_{\Theta_{1}}$ in $M_{\Theta_{2}}$.

Lemma 6.1. Let $P_{\Theta}$, given by $\Theta \subset \Delta_{0}$, be any maximal $\theta$-split $\Delta_{0}$-standard parabolic subgroup. Let $w \in\left[W_{\Theta} \backslash W_{0} / W_{\Omega}\right]$ such that $M_{\Theta} \cap{ }^{w} M_{\Omega}=M_{\Theta \cap w \Omega}$ is a proper Levi subgroup of ${ }^{w} M_{\Omega}=M_{w \Omega}$. We have the containment

$$
S_{\Theta}^{-} \backslash S_{\Theta}^{1} S_{\Delta_{0}} \subset A_{\Theta \cap w \Omega}^{-w \Omega} \backslash A_{\Theta \cap w \Omega}^{1} A_{w \Omega} .
$$

Recall that $S_{\Theta}^{1}=S_{\Theta}\left(\Theta_{F}\right)$ and $A_{\Theta \cap w \Omega}^{1}=A_{\Theta \cap w \Omega}\left(\Theta_{F}\right)$. Let $P=M N$ be a maximal $\theta$-split parabolic subgroup of $G$. By Lemma 4.3, there exists $g \in\left(\mathbf{H T}_{0}\right)(F)$ such that $P=g P_{\Theta} g^{-1}$, where $P_{\Theta}$ is a $\Delta_{0}$-standard maximal $\theta$-split parabolic subgroup. We may take $S_{M}=g S_{\Theta} g^{-1}$ and then we have $S_{M}^{-}=g S_{\Theta}^{-} g^{-1}$. Let $y \in P \backslash G / Q$, given by $y=g w \gamma$, where $w \in\left[W_{\Theta} \backslash W_{0} / W_{\Omega}\right]$. We observe that ${ }^{y} L=g\left(M_{w \Omega}\right) g^{-1}$. In particular, $M \cap{ }^{y} L=g\left(M_{\Theta \cap w \Omega}\right) g^{-1}$ and $A_{M \cap \cap^{y} L}=g\left(A_{\Theta \cap w \Omega}\right) g^{-1}$. The dominant part of the torus $A_{M \cap^{y} L}$ will be denoted by $A_{M \cap^{y} L}^{-y^{y}}$ and is determined by the simple roots $g w \Omega$ of the maximal $(\theta, F)$-split torus ${ }^{g} A_{0}$ in ${ }^{y} L$.

Lemma 6.2. Let $P=M N$ be any maximal $\theta$-split parabolic subgroup of $G$ with $\theta$-stable Levi $M$ and unipotent radical $N$. Choose a maximal subset $\Theta$ of $\Delta_{0}$ and an element $g \in\left(\mathbf{H T}_{0}\right)(F)$ such that $P=g P_{\Theta} g^{-1}$. Let $y=$ gw $\gamma \in P \backslash G / Q$, where $w \in\left[W_{\Theta} \backslash W_{0} / W_{\Omega}\right]$, such that $M \cap{ }^{y} L$ is a proper Levi subgroup of ${ }^{y} L$. Then we have the containment

$$
S_{M}^{-} \backslash S_{M}^{1} S_{G} \subset A_{M \cap^{y} L}^{-y^{y}} \backslash A_{M \cap^{y} L}^{1} A_{y_{L}} .
$$

Proof. The $(\theta, F)$-split component $S_{\Theta}$ of $M_{\Theta}$ is equal to its $F$-split component $A_{\Theta}$; moreover, we have that $S_{M}=A_{M}$. We also have that $S_{M}^{-}=$ $g S_{\Theta}^{-} g^{-1}$ and $S_{M}^{1}=g S_{\Theta}^{1} g^{-1}$; moreover, since $S_{G}=S_{\Delta_{0}}$ is central in $G$ we obtain

$$
S_{M}^{-} \backslash S_{G} S_{M}^{1}=g\left(S_{\Theta}^{-}\right) g^{-1} \backslash S_{G} g\left(S_{\Theta}^{1}\right) g^{-1} .
$$

By Lemma 6.1, we have that

$$
S_{\Theta}^{-} \backslash S_{\Theta}^{1} S_{\Delta_{0}} \subset A_{\Theta \cap w \Omega}^{-w \Omega} \backslash A_{\Theta \cap w \Omega}^{1} A_{w \Omega} .
$$

By the equality in (6.3), it suffices to show that

$$
A_{M \cap^{y} L}^{-y^{y}} \backslash A_{M \cap y}^{1} A_{y} L=g\left(A_{\Theta \cap w \Omega}^{-w \Omega}\right) g^{-1} \backslash g\left(A_{\Theta \cap w \Omega}^{1}\right) g^{-1} g\left(A_{w \Omega}\right) g^{-1} .
$$

Indeed, if (6.4) holds, then we have

$$
\begin{aligned}
S_{M}^{-} \backslash S_{G} S_{M}^{1} & =g S_{\Theta}^{-} g^{-1} \backslash S_{G} g S_{\Theta}^{1} g^{-1} \\
& \subset g\left(A_{\Theta \cap w \Omega}^{-w \Omega}\right) g^{-1} \backslash g\left(A_{\Theta \cap w \Omega}^{1}\right) g^{-1} g\left(A_{w \Omega}\right) g^{-1} \quad \text { Lemma 6.1) } \\
& =A_{M \cap^{y} L}^{-y_{L}} \backslash A_{M \cap^{y} L^{1} A_{L}}^{1},
\end{aligned}
$$

as claimed. The truth of (6.4) immediately follows from how we determine the dominant part of $A_{M \cap^{y} L}$. As above, we have that $M \cap{ }^{y} L=$ 
$g\left(M_{\Theta \cap w \Omega}\right) g^{-1}$ and $A_{M \cap^{y} L}=g\left(A_{\Theta \cap w \Omega}\right) g^{-1}$. Moreover, $A_{M \cap^{y} L}^{1}=g\left(A_{\Theta \cap w \Omega}^{1}\right) g^{-1}$. Given a root $\alpha \in \Phi_{0}$ we obtain a root $g \alpha$ of ${ }^{g} A_{0}$ in $G$ by setting $g \alpha=$ $\alpha \circ \operatorname{Int} g^{-1}$, as usual. Explicitly, we have that

$$
A_{M \cap^{y} L}^{-y}=\left\{a \in A_{M \cap^{y} L}:|g \beta(a)| \leq 1, \beta \in w \Omega \backslash \Theta \cap w \Omega\right\} .
$$

In fact, we have that $M \cap{ }^{y} L$ is determined (as Levi subgroup of ${ }^{y} L$ ) by the simple roots $g(\Theta \cap w \Omega) \subset g w \Omega$ of ${ }^{g} A_{0}$ in ${ }^{y} L=g w M_{\Omega}$. It is immediate that

$$
A_{M \cap^{y} L}^{-y}=g\left(A_{\Theta \cap w \Omega}^{-w \Omega}\right) g^{-1},
$$

from which (6.4) follows, completing the proof of the lemma.

Acknowledgements. I would like to thank my doctoral advisor Fiona Murnaghan for her support and guidance throughout the initial work on this project. Thank you to Raphaël Beuzart-Plessis for providing copies of his 2017 Cours Peccot lecture notes and for his generous explanations. I would also like to thank the referee for their valuable comments and, in particular, for the suggested improvements to Propositions 5.12 and 5.15. Finally, thank you to the editor for directing my attention to the forthcoming work of Beuzart-Plessis and suggesting the inclusion of Section 5.4.

\section{REFERENCES}

1. James Arthur and Laurent Clozel, Simple algebras, base change, and the advanced theory of the trace formula, Annals of Mathematics Studies, vol. 120, Princeton University Press, Princeton, NJ, 1989. MR 1007299

2. I. N. Bernstein and A. V. Zelevinsky, Representations of the group $G L(n, F)$, where $F$ is a local non-Archimedean field, Uspehi Mat. Nauk 31 (1976), no. 3(189), 5-70. MR 0425030 (54 \#12988)

3. Induced representations of reductive $p$-adic groups. I, Ann. Sci. École Norm. Sup. (4) 10 (1977), no. 4, 441-472. MR 0579172 (58 \#28310)

4. Raphaël Beuzart-Plessis, Factorisations de périodes et formules de plancherel, Unpublished notes; Cours Peccot, 2017.

5. François Bruhat, Distributions sur un groupe localement compact et applications à l'étude des représentations des groupes §-adiques, Bull. Soc. Math. France 89 (1961), 43-75. MR 0140941

6. William Casselman, Introduction to the theory of admissible representations of $p$-adic reductive groups, Unpublished manuscript, draft prepared by the Séminaire Paul Sally, 1995, Available at www.math.ubc.ca/ cass/research/publications.html

7. François Digne and Jean Michel, Groupes réductifs non connexes, Ann. Sci. École Norm. Sup. (4) 27 (1994), no. 3, 345-406. MR 1272294

8. Brooke Feigon, Erez Lapid, and Omer Offen, On representations distinguished by unitary groups, Publ. Math. Inst. Hautes Études Sci. (2012), 185-323. MR 2930996

9. I. M. Gel'fand and D. A. Kajdan, Representations of the group $\mathrm{GL}(n, K)$ where $K$ is a local field, Lie groups and their representations (Proc. Summer School, Bolyai János Math. Soc., Budapest, 1971), Halsted, New York, 1975, pp. 95-118. MR 0404534 (53 \#8334)

10. Maxim Gurevich and Omer Offen, A criterion for integrability of matrix coefficients with respect to a symmetric space, J. Funct. Anal. 270 (2016), no. 12, 4478-4512. MR 3490774 
11. Jeffrey Hakim and Fiona Murnaghan, Two types of distinguished supercuspidal representations, Int. Math. Res. Not. IMRN (2002), no. 35, 1857-1889. MR 1920643 (2003f:22023)

12. A. G. Helminck and G. F. Helminck, A class of parabolic k-subgroups associated with symmetric k-varieties, Trans. Amer. Math. Soc. 350 (1998), no. 11, 4669-4691. MR 1443876 (99g:20082)

13. A. G. Helminck and S. P. Wang, On rationality properties of involutions of reductive groups, Adv. Math. 99 (1993), no. 1, 26-96. MR 1215304 (94d:20051)

14. Aloysius G. Helminck, Algebraic groups with a commuting pair of involutions and semisimple symmetric spaces, Adv. in Math. 71 (1988), no. 1, 21-91. MR 960363

15. H. Jacquet and K. F. Lai, A relative trace formula, Compositio Math. 54 (1985), no. 2, 243-310. MR 783512

16. Hervé Jacquet, Factorization of period integrals, J. Number Theory 87 (2001), no. 1, 109-143. MR 1816039

17. functions and applications: progress and prospects, Ohio State Univ. Math. Res. Inst. Publ., vol. 11, de Gruyter, Berlin, 2005, pp. 257-272. MR 2192826

18. _ Distinction by the quasi-split unitary group, Israel J. Math. 178 (2010), 269324. MR 2733072

19. Hervé Jacquet, Erez Lapid, and Jonathan Rogawski, Periods of automorphic forms, J. Amer. Math. Soc. 12 (1999), no. 1, 173-240. MR 1625060 (99c:11056)

20. Shin-Ichi Kato and Keiji Takano, Subrepresentation theorem for p-adic symmetric spaces, Int. Math. Res. Not. IMRN (2008), no. 11. MR 2428854

21. _ Square integrability of representations on p-adic symmetric spaces, J. Funct. Anal. 258 (2010), no. 5, 1427-1451. MR 2566307

22. Nathalie Lagier, Terme constant de fonctions sur un espace symétrique réductif padique, J. Funct. Anal. 254 (2008), no. 4, 1088-1145. MR 2381204 (2009d:22013)

23. Erez M. Lapid and Jonathan D. Rogawski, Periods of Eisenstein series: the Galois case, Duke Math. J. 120 (2003), no. 1, 153-226. MR 2010737

24. Fiona Murnaghan, Regularity and distinction of supercuspidal representations, Harmonic analysis on reductive, $p$-adic groups, Contemp. Math., vol. 543, Amer. Math. Soc., Providence, RI, 2011, pp. 155-183. MR 2798427

25. Distinguished positive regular representations, Special Issue of the Bulletin of the Iranian Mathematical Society, in Honor of Professor Freydoon Shahidi's 70th birthday 43 (2017), no. 4, 291-311.

26. Alain Robert, Introduction to the representation theory of compact and locally compact groups, London Mathematical Society Lecture Note Series, vol. 80, Cambridge University Press, Cambridge-New York, 1983. MR 690955

27. Alan Roche, The Bernstein decomposition and the Bernstein centre, Ottawa lectures on admissible representations of reductive $p$-adic groups, Fields Inst. Monogr., vol. 26, Amer. Math. Soc., Providence, RI, 2009, pp. 3-52. MR 2508719

28. Yiannis Sakellaridis and Akshay Venkatesh, Periods and harmonic analysis on spherical varieties, Astérisque 396 (2017), viii+360.

29. Jerrod Manford Smith, Relative discrete series representations for two quotients of p-adic $\mathbf{G L}_{n}$, Canad. J. Math. (2018), doi.

30. _ Construction of relative discrete series representations for p-adic $\mathbf{G L}_{n}$, Ph.D. thesis, University of Toronto, June 2017, hdl].

31. A. V. Zelevinsky, Induced representations of reductive p-adic groups. II. On irreducible representations of GL(n), Ann. Sci. École Norm. Sup. (4) 13 (1980), no. 2, 165-210. MR 584084 (83g:22012)

University of Maine, Department of Mathematics \& Statistics, Orono, Maine E-mail address: jerrod.smith@maine.edu 